\title{
Discrete symmetries, weak coupling conjecture and scale separation in AdS vacua
}

\author{
Ginevra Buratti, José Calderón, Alessandro Mininno and Angel M. Uranga \\ Instituto de Física Teórica IFT-UAM/CSIC, \\ C/ Nicolás Cabrera 13-15, Campus de Cantoblanco, 28049 Madrid, Spain \\ E-mail: ginevra.buratti@uam.es, j.calderon.infante@csic.es, \\ alessandro.mininno@uam.es, angel.uranga@csic.es
}

ABSTRACT: We argue that in theories of quantum gravity with discrete gauge symmetries, e.g. $\mathbf{Z}_{k}$, the gauge couplings of $\mathrm{U}(1)$ gauge symmetries become weak in the limit of large $k$, as $g \rightarrow k^{-\alpha}$ with $\alpha$ a positive order 1 coefficient. The conjecture is based on black hole arguments combined with the Weak Gravity Conjecture (or the BPS bound in the supersymmetric setup), and the species bound. We provide explicit examples based on type IIB on $\mathrm{AdS}_{5} \times \mathbf{S}^{5} / \mathbf{Z}_{k}$ orbifolds, and M-theory on $\mathrm{AdS}_{4} \times \mathbf{S}^{7} / \mathbf{Z}_{k}$ ABJM orbifolds (and their type IIA reductions). We study $\mathrm{AdS}_{4}$ vacua of type IIA on CY orientifold compactifications, and show that the parametric scale separation in certain infinite families is controlled by a discrete $\mathbf{Z}_{k}$ symmetry for domain walls. We accordingly propose a refined version of the strong AdS Distance Conjecture, including a parametric dependence on the order of the discrete symmetry for 3 -forms.

KEYwords: Discrete Symmetries, Flux compactifications, Superstring Vacua, Supersymmetric Effective Theories

ArXiv ePrint: 2003.09740 


\section{Contents}

1 Introduction and conclusions 1

2 The $\mathrm{Z}_{k}$ weak coupling conjecture 4

2.1 A black hole argument 4

2.1.1 Review of some mass bound derivations 4

2.1.2 The $\mathbf{Z}_{k}$ weak gravity conjecture 5

2.1.3 The $\mathbf{Z}_{k}$ weak coupling conjecture 6

$\begin{array}{lll}2.2 & \text { Distance conjectures } & 7\end{array}$

$3 \mathrm{AdS}_{5} \times \mathrm{S}^{5}$ orbifolds $\quad 8$

4 M-theory orbifolds and ABJM

4.1 M-theory on $\mathrm{AdS}_{4} \times \mathbf{S}^{7} / \mathbf{Z}_{k} \quad 12$

$\begin{array}{lll}4.2 & \text { Type IIA description of ABJM vacua } & 14\end{array}$

5 Discrete 3-form symmetries and scale separation in AdS solutions 16

$\begin{array}{lll}5.1 & \text { Review of scaling } \mathrm{AdS}_{4} \text { vacua with scale separation } & 17\end{array}$

$\begin{array}{ll}5.2 \text { The discrete 3-form symmetry } & 19\end{array}$

5.3 Scaling relations for moduli from discrete symmetries 21

5.4 Discrete symmetries and scale separation 23

$\begin{array}{lll}\text { 5.4.1 Vacuum energy from domain walls } & 24\end{array}$

5.4.2 Warm-up examples: no scale separation 24

5.4.3 Revisiting the scale separation in type IIA CY flux compactifications 26

$\begin{array}{ll}\text { A Species bound for extremal black holes } & 27\end{array}$

$\begin{array}{ll}\text { B Discrete symmetries in intersecting brane models } & 28\end{array}$

C Gauge couplings in type IIA CY compactifications 30

D Junction conditions for AdS vacua 32

\section{Introduction and conclusions}

By now there is a substantial amount of swampland conjectures constraining effective field theories to be compatible with Quantum Gravity [1-6] (see [7, 8] for reviews). They have led to interesting insights into phenomenological applications of string theory models.

Interestingly, many of these works focus on the properties of continuous gauge symmetries, whereas far fewer results have been obtained to constrain discrete symmetries (for 
some results, see [9-11], and also [12]), and mostly focus on the constrain that global discrete symmetries, just like global continuous symmetries, are forbidden in quantum gravity (see [13-19] for early literature). Discrete gauge symmetries are an interesting area with exciting applications in BSM phenomenology and string model building [20-26]. The scarcity of swampland constraints on them is partially explained by the fact that discrete symmetries lack long-range fields or tunable parameters like coupling constants, so there are less handles to quantitatively constrain their properties or their impact on other quantities of the theory.

In this work, we overcome this difficulty by considering theories with both discrete and continuous gauge symmetries, and uncover interesting quantitative links among them. For simplicity we focus on abelian $\mathbf{Z}_{k}$ and $\mathrm{U}(1)$ symmetries. In theories with a $\mathrm{U}(1)$ gauge symmetry, considerations about evaporation of charged black holes lead to the Weak Gravity Conjecture [3], by demanding that the black hole should remain (sub)extremal throughout the process. To put it simply, considering an extremal black hole with $M=g Q$ (in Planck units), the theory must contain particles with mass $m$ and charge $q$, with $m \leq g q$, such that the black hole can decay without becoming super-extremal. This is the Weak Gravity Conjecture (WGC). The marginal case in which the Weak Gravity Conjecture particles saturate the inequality $m=q$ has been further proposed to correspond to supersymmetric situations, in which it often corresponds to a BPS bound.

If the theory enjoys a further $\mathbf{Z}_{k}$ discrete gauge symmetry, one can consider any such classical black hole solution and endow it with discrete $\mathbf{Z}_{k}$ charge, with no change in the classical solution, as this charge does not source long-range fields (see e.g. [27], and also [28] for a recent perspective), and study their decay as in the WGC. In particular, we may consider extremal black holes carrying $\mathbf{Z}_{k}$ charge and derive a striking result, the $\mathbf{Z}_{k}$ Weak Coupling Conjecture (WCC) which schematically is the statement that in a theory with a discrete $\mathbf{Z}_{k}$ gauge symmetry and a $\mathrm{U}(1)$ gauge symmetry with coupling $g$, the gauge coupling scales as $g \sim k^{-\alpha}$ for large $k$, with $\alpha$ a positive order 1 coefficient.

The derivation and some qualifications on this statement are discussed in section 2. In particular, we also relate this statement with diverse versions of swampland distance conjectures.

As we will see, the derivation is most precise in the supersymmetric case, in which the WGC bound saturates, but we believe it holds far more generally, as we will illustrate in concrete string theory examples. In particular, in section 3 we study $\mathrm{AdS}_{5} \times \mathbf{S}^{5} / \mathbf{Z}_{k}$ vacua (and generalizations to general toric ${ }^{1}$ theories $\mathrm{AdS}_{5} \times \mathbf{X}_{5} / \mathbf{Z}_{k}$ ), in which there is a discrete Heinsenberg group $\mathbf{H}_{k}$, associated to torsion classes in $\mathbf{S}^{5} / \mathbf{Z}_{k}$ [29-31]. This is generated by elements $A, B$, each generating a $\mathbf{Z}_{k}$ symmetry, with commutation relations $A B=C B A$, with $C$ a central element. In the effective 5 d theory (namely at scales below the KK scale, and thus at long distance compared with the AdS radius as well) there is at least one $\mathrm{U}(1)$ gauge symmetry, corresponding to the R-symmetry of the holographic dual SCFT, whose coupling, as we show, obeys the WCC. In addition, for $\mathbf{S}^{5} / \mathbf{Z}_{k}$, and in fact for any toric

\footnotetext{
${ }^{1}$ By toric, in this context we mean that the CY3 obtained as the real cone over the Sasaki-Einstein $5 \mathrm{~d}$ variety, is toric.
} 
theory $\mathbf{X}_{5} / \mathbf{Z}_{k}$, there are two additional U(1)'s (the mesonic global symmetries in the dual SCFT), which also satisfy the WCC.

In section 4 we discuss an analogous exercise in $4 \mathrm{~d}$ by considering in section 4.1 the case of M-theory on $\mathrm{AdS}_{4} \times \mathbf{S}^{7} / \mathbf{Z}_{k}$, which provides the gravity dual to the ABJM theories [32]. The $\mathrm{U}(1)$ symmetry corresponds to an isometry of the internal space, and the discrete symmetry is also related to torsion classes in $\mathbf{S}^{7} / \mathbf{Z}_{k}$, although it has an intricate structure not reducible to just $\mathbf{Z}_{k}$. This is further clarified using the type IIA perspective in section 4.2 , in which the discrete gauge symmetry is shown to have order $k^{2}+N^{2}$, and the $\mathrm{U}(1)$ symmetry is a linear combination of different RR p-form gauge symmetries, with a second linear combination that is massive due to a Stückelberg coupling. We discuss these systems and show how the corresponding WCC is duly satisfied.

In section 5 we turn to exploiting these considerations in theories in which the $\mathbf{Z}_{k}$ charged objects are not particles (or their dual objects, e.g. strings in 4d), but rather $4 \mathrm{~d}$ domain walls. In particular, we consider the type IIA $\mathrm{AdS}_{4}$ vacua obtained in $\mathrm{CY}$ orientifold compactification with NSNS and RR fluxes. In section 5.1 we review a class of compactifications with fluxes scaling with a parameter $k$, shown in [33] to have parametric scale separation controlled by $k$. These vacua would violate the strong AdS Distance Conjecture proposed in [34], an issue on which our analysis sheds important insights. In section 5.2 we show that these systems are higher p-form analogues to the type IIA vacua of section 4.2 , with a continuous 3 -form symmetry arising from a massless linear combination, and the discrete symmetry arising from a second linear combination made massive by a 3-form Stückelberg mechanism (see [35, 36], also [24]), also called Dvali-Kaloper-Sorbo (DKS) mechanism. In section 5.3 we discuss the role of the discrete $\mathbf{Z}_{k}$ symmetry in fixing the scaling of the moduli with $k$. In section 5.4 we use tensions of BPS domain walls to recover the vacuum energy scalings, and show that AdS vacua with trivial 3-form discrete symmetry have no scale separation, while the above scaling family of AdS vacua with a non-trivial 3-form discrete symmetry displays scale separation controlled by $k$, as follows. The scale separation relation between the KK scale $m_{K K}$ and the $4 \mathrm{~d}$ cosmological constant $\Lambda$ is given by the species bound

$$
\Lambda=\frac{m_{\mathrm{KK}}^{2}}{k} .
$$

We accordingly formulate the following $\mathbf{Z}_{k}$ Refined Strong $\mathrm{AdS}_{4}$ Distance Conjecture: in supersymmetric $\mathrm{AdS}_{4}$ vacua with a discrete symmetry associated to $\mathbf{Z}_{k}$-charged domain walls, the ratio between the KK scale and $\Lambda$ is $m_{\mathrm{KK}} \sim(k \Lambda)^{1 / 2}$.

This provides an underlying rationale for the seeming violation of the strong ADC by the family of scaling AdS solutions in type IIA vacua with field strength fluxes. It would be interesting to test it in other setups, and even exploit it in applications to holography.

Our work is an important step in understanding the nature of discrete gauge symmetries in quantum gravity, and their non-trivial interplay with continuous gauge symmetries. As in other swampland constraints, although the arguments for the $\mathbf{Z}_{k}$-WCC are admittedly heuristic, there is a substantial amount of evidence from concrete, very rigorous, string vacua supporting it. We have argued that discrete symmetries for 3 -forms play an 
important role in the problem of scale separation, and provided a rationale to embed it in a refined AdS Distance Conjecture. We thus expect they may be relevant in other swampland criteria, like the de Sitter constraint. We hope to report on these topics in the near future.

Note: as we were finishing writing this paper, ref. [37] appeared, which studies scale separation in type IIA AdS vacua, albeit from a different perspective (note also [38], appeared shortly after our work). It would be interesting to explore the relation between the two approaches.

\section{The $\mathrm{Z}_{k}$ weak coupling conjecture}

In this section we consider theories of quantum gravity with discrete and continuous gauge symmetries. For simplicity we focus on a $\mathbf{Z}_{k}$ discrete symmetry and a $\mathbf{U}(1)$ gauge symmetry. Generalizations to multiple U(1)'s and discrete groups could be worked out similarly. Notice that throughout the paper we are interested in the properties of the theory at large $k$, hence many of our expressions should be regarded as the leading approximation in a $1 / k$ expansion.

\subsection{A black hole argument}

For concreteness we focus on $4 \mathrm{~d}$ theories, although the results extend to other dimensions (as we will see e.g. in the examples of section 3). The strategy is to use black hole evaporation as a guiding principle to derive new swampland constraints, as we now review in two familiar situations.

\subsubsection{Review of some mass bound derivations}

Let us briefly recall one such derivation for the Weak Gravity Conjecture (WGC) [3]. The idea is to consider extremal black holes, with mass $M$ and charge $Q$, satisfying $M=$ $g Q M_{p}$, where $g$ is the $\mathrm{U}(1)$ gauge coupling (in units in which the minimal charge is 1 ). Requiring the decay of such extremal black holes, while preventing them from becoming super-extremal, leads to the familiar statement of the Weak Gravity Conjecture, namely that there must exist some particle in the theory with mass $m$ and charge $q$ such that

$$
m \leq g q M_{p}
$$

There are different versions of the WGC (see [8] for a review with references), including the lattice [39] and sublattice [40] versions, but we stick to the basic one above.

Let us consider a black hole (possibly charged under the $\mathrm{U}(1)$ or not), carrying a discrete $\mathbf{Z}_{k}$ charge. The analysis now follows [41]. Even though this is a gauge symmetry, it does not have long-range fields, so it does not affect the classical black hole solution, neither its evaporation in the semiclassical approximation, which thus does not allow to eliminate the $\mathbf{Z}_{k}$ charge. Since we are interested in the large $k$ behavior, this would lead to a too large number of remnants. Hence, when the black hole radius reaches some cutoff value $\Lambda^{-1}$ it starts peeling off its $\mathbf{Z}_{k}$ charge. If we denote by $m$ the mass of the $\mathbf{Z}_{k}$ charged 
particles, the mass of the black hole at the cutoff scale should suffice to emit $\mathcal{O}(k)$ of such particles, that is

$$
M_{p}^{2} \Lambda^{-1} \gtrsim k m
$$

The cutoff radius is intuitively of the order of the inverse mass of the emitted particle, hence we consider $\Lambda \sim \beta m$, with $\beta$ some unknown coefficient encoding model dependent information about the black hole and its evaporation process. Consequently, we obtain

$$
m^{2} \lesssim \frac{M_{p}^{2}}{k}
$$

This is often known as the species bound [41], although in the present context $k$ does not correspond to the number of species, rather it relates to the order of the discrete symmetry. ${ }^{2}$

Keeping in mind the unknown factors in the discussion, we take the above relation as controlling the scaling of suitable $\mathbf{Z}_{k}$ charged particles in the limit of large $k$. Namely, there must exist some $\mathbf{Z}_{k}$ charged particle whose mass must scale as $m \lesssim k^{-1 / 2} M_{p}$.

In the following, we will apply this constraint to black holes charged under continuous U(1) symmetries. One may worry that the derivation in [41] did not include such charges, i.e. it implicitly assumed Schwarzschild black holes. However, there are analogous arguments for charged (in fact extremal) black holes in theories with $\mathrm{U}(1)$ gauge groups, leading to identical results, as we discuss in appendix A. Hence for practical purposes we may continue with the above simple picture.

\subsubsection{The $\mathrm{Z}_{k}$ weak gravity conjecture}

In the above discussion, the mass of the $\mathbf{Z}_{k}$ particle we are constraining is thought of as the lightest one. However, in the following we argue that we can use a similar argument to constrain not only the lightest $\mathbf{Z}_{k}$ charge particle, but also the one with smallest ratio $q / m$ between its $\mathrm{U}(1)$ charge and its mass. Namely, the Weak Gravity Conjecture particles.

Consider an extremal black hole with mass $M$ and charge $Q$, and endow it with a large $\mathbf{Z}_{k}$ charge. The black hole can try to peel off its $\mathbf{Z}_{k}$ charge by emitting $\mathbf{Z}_{k}$ charged particles, but this would decrease its mass while keeping its charge fixed, thus becoming super-extremal. The simplest way to prevent this is that there exist some $\mathbf{Z}_{k}$ charged particle which is also charged under the $\mathrm{U}(1)$ with charge $q$, and such that it satisfies the WGC bound $m \leq g q M_{p}$. In other words, the simplest resolution is that the WGC particles carry $\mathbf{Z}_{k}$ charge. We may dub this result as the $\mathbf{Z}_{k}$ Weak Gravity Conjecture.

This is a remarkable result, but is actually a little bit of an overstatement. It may well happen that the WGC particles are neutral and do not saturate the WGC bound, and the evaporation of the black hole by emission of WGC particles makes it sufficiently sub-extremal so as to be able to subsequently emit enough $\mathbf{Z}_{k}$ charged particles (not obeying the WGC bound) to peel off its discrete charge without ever getting super-extremal.

\footnotetext{
${ }^{2}$ Actually, to account for the fact that the particle needs not be minimally charged under $\mathbf{Z}_{k}$, we should point out that the role of $k$ above should actually be played by the number of emitted particles. Hence the factor appearing in relations like (2.3) may differ from the order of the discrete group by a factor of the particle charge, see some examples in sections 4,5 .
} 
Interestingly, notice that this is only possible if the WGC particles satisfy the strict WGC bound, not the equality, and hence, according to the extended WGC version in [4], it is possible only in non-supersymmetric theories. Thus our derivation above is strictly valid in the supersymmetric setup, and in our examples we will indeed focus on supersymmetric examples. We however still consider the argument as interestingly compelling also in nonsupersymmetric models, and hence keep an open mind about its general validity, and that of its implications, to which we turn.

\subsubsection{The $\mathrm{Z}_{k}$ weak coupling conjecture}

The fact that the WGC particles, whose defining feature has to do with the U(1) gauge symmetry, know about the $\mathbf{Z}_{k}$ symmetry implies that there are cross constraints among the $\mathrm{U}(1)$ and the $\mathbf{Z}_{k}$ symmetry. Indeed, let us consider a relaxed version of the $\mathbf{Z}_{k}$ bound (2.3), by stating that the $\mathbf{Z}_{k}$ charged particles involved in the black hole decay should have mass scaling as

$$
m \sim k^{-\alpha} M_{p}
$$

with $\alpha$ an order 1 coefficient, obeying some bound $\alpha \geq 1 / 2$ to satisfy (2.3). On the other hand, the particles that extremal black holes use to peel off their $\mathbf{Z}_{k}$ charge are WGC particles, hence obey

$$
m \sim g q M_{p} .
$$

We thus obtain that the gauge coupling of the $\mathrm{U}(1)$ must depend on $k$ and should become weak fast enough in the large $k$ limit, as

$$
g \sim k^{-\alpha} .
$$

We thus propose this to be a general swampland constraint, as follows:

$\mathbf{Z}_{k}$ weak coupling conjecture. In a quantum gravity theory with a discrete $\mathbf{Z}_{k}$ gauge symmetry and $a \mathrm{U}(1)$ gauge symmetry with coupling $g$, the gauge coupling scales as $g \sim k^{-\alpha}$ for large $k$, with $\alpha$ a positive order 1 coefficient.

We note that, in the case of multiple $\mathrm{U}(1)$ gauge symmetries, a similar $\mathrm{BH}$ argument leads to a $\mathbf{Z}_{k}$ Weak Coupling Conjecture for any rational direction in charge space, much in the spirit of the WGC for multiple U(1)'s [42]. Since the gauge coupling of any linear combination follows from those in some basis in the charge lattice, in this case it suffices that the couplings of these independent $\mathrm{U}(1)$ obey the $\mathbf{Z}_{k}$ Weak Coupling Conjecture. We also note that in the case of multiple discrete symmetries, the conjecture applies to each discrete symmetry independently.

The above intertwining between the properties of discrete and continuous symmetries is completely unexpected from the viewpoint of the low energy effective field theory, where these parameters are uncorrelated and would seem to be completely free choices. As with other swampland constraints, it is amusing that quantum gravity manages to impose its own plans. 
A simple illustration of how this interplay works in intersecting brane modes is discussed at the heuristic level in appendix B. More concrete examples will follow in the upcoming sections.

\subsection{Distance conjectures}

Before moving to concrete examples, it is interesting to explore the relation between the $\mathbf{Z}_{k}$ WCC and the Swampland Distance Conjectures (SDC). The WCC states that gauge couplings scale to zero for large $k$, thus approaching a global symmetry and hence presumably leading to the appearance of a tower of states becoming light.

An intuitive picture of this implication is as follows. Consider a $4 \mathrm{~d}$ version of the $\mathbf{Z}_{k}$ WCC with $g \sim k^{-\alpha}$. For simplicity, and following many examples in string theory we consider $g$ to belong to a complex modulus

$$
S=\frac{1}{g^{2}}+i \theta
$$

and assume a Kähler potential

$$
K(S, \bar{S})=-\log (S+\bar{S}) .
$$

In this moduli space, the distance as a function of $s=\operatorname{Re} S$ as one approaches infinity reads

$$
d \sim \int \frac{d s}{s} \sim \log s .
$$

The SDC states that there is a tower of states becoming light as $s \rightarrow \infty$ with masses

$$
m_{\mathrm{tw}} \sim M_{p} e^{-\gamma d},
$$

with $\gamma$ an order 1 coefficient, for $d$ measured in Planck units. In our case we have

$$
m_{\mathrm{tw}} \sim M_{p} k^{-\frac{1}{2} \alpha \gamma} .
$$

Hence there is a $\mathbf{Z}_{k}$ Distance Conjecture stating that there is a tower of states with masses becoming light as a negative power of $k$. This is just a re-derivation of the 'species' bound cutoff [41].

In fact, the above argument where $g$ is dealt with as a modulus going to infinite distance in moduli space does not correspond to the general $\mathbf{Z}_{k}$ WCC, since at least some of the gauge couplings may not correspond to fundamental moduli. For instance, consider the intersecting brane toy model in appendix B. There, the moduli remain at fixed location in moduli space, and we instead change the discrete wrapping numbers for some D-branes. Hence, the origin of the tower should be a different one, as is easily argued. In a configuration in which one stack of branes has wrappings scaling with $k$, the angles between that stack of branes and others will scale as $\theta \sim k^{-1}$ (to see that, consider e.g. the cycles $(1,0)$ and $(k, 1)$ in a rectangular $\mathbf{T}^{2}$ with radii $\left(R_{1}, R_{2}\right)$. They have intersection angle $\theta$ with $\tan \theta=k^{-1} R_{2} / R_{1}$, hence $\left.\theta \sim k^{-1}\right)$. As discussed in [43, 44] there is a tower of string states with masses given by

$$
m_{\mathrm{tw}}^{2} \sim M_{s} \theta \sim k^{-1} .
$$

This again nicely reproduces the 'species' bound cutoff. 


\section{$3 \quad \mathrm{AdS}_{5} \times \mathrm{S}^{5}$ orbifolds}

In this section we consider type IIB string theory on $\mathrm{AdS}_{5} \times \mathbf{S}^{5} / \mathbf{Z}_{k}$. The discussion can be easily extended to general toric orbifold theories $\mathrm{AdS}_{5} \times \mathbf{X}_{5} / \mathbf{Z}_{k}$, but the 5 -sphere case will suffice to illustrate the main points. We study general $\mathbf{Z}_{k}$ actions compatible with supersymmetry, namely acting as $\mathrm{SU}(3)$ in the underlying $\mathbf{C}^{3}$. We also note that, although these vacua do not display scale separation, we may discuss the 5 d physics essentially in the same sense as in the AdS/CFT correspondence, whose dictionary and results we use freely in this section. Moreover, our final statement involves gauge couplings for U(1) symmetries, which can be observed at arbitrarily long distances, in particular at energies well below the KK scale.

As pioneered in [29] (see also [30, 45-47] for other examples) and generalized in [31], there is a discrete gauge symmetry in the $\mathrm{AdS}_{5}$ theory, corresponding to the discrete Heisenberg group $\mathbf{H}_{k}$. This is defined by two non-commuting $\mathbf{Z}_{k}$ symmetries generated by $A, B$ (hence $A^{k}=1, B^{k}=1$ ) satisfying

$$
A B=C B A
$$

with $C$ a central element (also generating a further $\mathbf{Z}_{k}$, and possibly mixing with other anomaly free baryonic U(1)'s, if present).

Generalizing [29], the particles charged under the discrete symmetry are D3-branes wrapped on torsion 3-cycles carrying non-trivial flat gauge bundles (discrete Wilson lines and 't Hooft loops). The minimally charged particle is obtained by wrapping the D3-brane on a maximal $\mathbf{S}^{3} / \mathbf{Z}_{k}$. We are interested in the mass of this particle, and in particular in its scaling with $k$. It is a simple exercise, as this is just analogous to a giant graviton in the parent $\mathrm{AdS}_{5} \times \mathbf{S}^{5}$ theory [48].

The D3-brane particle mass computation. In the KK reduction from $10 \mathrm{~d}$ to $5 \mathrm{~d}$, the 5 d Planck mass $M_{p, 5}$ in terms of the string scale is

$$
M_{p, 5}^{3}=\frac{M_{s}^{8} R^{5}}{g_{s}^{2} k}
$$

We are ignoring numerical factors e.g. in the volume of $\mathbf{S}^{5}$. Above, $R$ is the curvature radius of $\mathbf{S}^{5}$, which is also the $\mathrm{AdS}_{5}$ radius. Note that in order to get a theory with $N$ units of RR 5-form flux over $\mathbf{S}^{5} / \mathbf{Z}_{k}$, the parent theory is the $\mathrm{AdS}_{5} \times \mathbf{S}^{5}$ solution corresponding to $N k$ D3-branes, and the usual relation between the radius $R$ and $N$ is modified to

$$
R^{4}=4 \pi\left(\alpha^{\prime}\right)^{2} g_{s} N k
$$

Hence

$$
R \sim M_{s}^{-1} g_{s}^{\frac{1}{4}} N^{\frac{1}{4}} k^{\frac{1}{4}}
$$

where we have dropped numerical factors. 
The mass $m$ of the D3-brane particle ${ }^{3}$ in $5 \mathrm{~d}$ is

$$
m=\frac{M_{s}^{4} R^{3}}{g_{s} k}
$$

We wish to express the mass in terms of the 5 d Planck scale. From (3.2) and (3.4) we get

$$
M_{s} \sim M_{p, 5} g_{s}^{\frac{1}{4}} N^{-\frac{5}{12}} k^{-\frac{1}{12}} \quad, \quad R \sim M_{p, 5}^{-1} N^{\frac{2}{3}} k^{\frac{1}{3}}
$$

Hence

$$
m \sim M_{p, 5} N^{\frac{1}{3}} k^{-\frac{1}{3}}
$$

Note that the $k$-dependence reproduces the 5 d version of the relation (2.3) [41]

$$
m^{3} \sim \frac{M_{p, 5}^{3}}{k} .
$$

This result fits nicely with the expectation for the mass of a particle charged under $\mathbf{Z}_{k}$.

Notice that, as mentioned in section 2.1, the coefficient in (3.8) is not necessarily the order of the discrete symmetry (which we recall is the Heisenberg group $\mathbf{H}_{k}$ ) but the number of particles emitted to peel off the black hole charge. We also note that the factor of $N$ in (3.7) is presumably related to the precise nature of the cutoff $\Lambda$ in the black hole argument in section 2.1.1. It would be interesting to explore this dependence in more detail, but we leave this for future work.

Comparison with the BPS formula and WCC. The above states are not the lightest carrying charges under the $\mathbf{Z}_{k}$ subgroups of the Heisenberg group. In fact, there are charged particle states arising from fundamental strings and D1-branes wrapped on torsion 1-cycles on the internal geometry. What is special about the above D3-brane particle states is that they are BPS. Just like giant gravitons in $\mathrm{AdS}_{5} \times \mathbf{S}^{5}$, they carry $N$ units of momentum along a maximal $\mathbf{S}^{1}$, determined by the $\mathbf{Z}_{k}$ action. In the $5 \mathrm{~d}$ theory, there is a $\mathrm{KK} \mathrm{U}(1)_{R}$, which is precisely the gravity dual of the R-symmetry of the holographic SCFT. In the SCFT, the D3-brane particle states are dibaryons of the form $\operatorname{det} \Phi_{i j}$, with $\Phi$ denoting a generic bifundamental chiral multiplet in the quiver gauge theory. It has R-charge $N$, and conformal dimension $\Delta=N$. Using the AdS/CFT dictionary, we then expect the masses of these particles to be given by

$$
m=\frac{N}{R}
$$

The fact that these states are BPS means that they should saturate the WGC conjecture bound, in other words, the BPS mass formula

$$
m=\left(g M_{p, 5}^{\frac{1}{2}}\right) N M_{p, 5} .
$$

\footnotetext{
${ }^{3}$ Notice that for our purposes it does not matter if we are in the string or Einstein frame, since this introduces factors that depend on dynamical fields, but does not change the scaling with $k$, which goes into the constant part (reference value).
} 
This is the standard $m=g Q$ in Planck units, with charge $q=N$ and $g$ being the gauge coupling of the $\mathrm{U}(1)$.

In these relations, there is no manifest dependence on $k$, which could be puzzling from the viewpoint of the black hole arguments. As we however know, the resolution is that, on these general grounds, the gauge coupling $g$ must scale with $k$, at large $k$, in particular

$$
g \sim k^{-\frac{1}{3}} .
$$

This is easily checked by computing the gauge coupling. In the KK reduction from $10 \mathrm{~d}$ to $5 \mathrm{~d}$, the prefactor of the gauge kinetic term is

$$
\frac{1}{g^{2}}=\frac{M_{s}^{8} R^{5}}{g_{s}^{2} k} R^{2}
$$

The first factor is just the $10 \mathrm{~d}$ prefactor times the volume of $\mathbf{S}^{5} / \mathbf{Z}_{k}$, and the $R^{2}$ comes from the rescaling of mixed components of the metric into dimensionful gauge field, such that charges are quantized in integers.

Using our above expressions, we get

$$
g \sim R^{-1} M_{p, 5}^{-\frac{3}{2}}
$$

which means

$$
g M_{p, 5}^{\frac{1}{2}}=N^{-\frac{2}{3}} k^{-\frac{1}{3}}
$$

So, in terms of this gauge coupling, the mass (3.7) turns into (3.10). Hence we recover a very explicit confirmation of our heuristic argument in section 2 .

Let us conclude with some general remarks.

- In addition to $\mathrm{U}(1)_{R}$ there are in general (in fact, for general toric theories) two extra mesonic U(1) symmetries, arising from isometries of the internal $5 \mathrm{~d}$ manifold. The direct computation of their $5 \mathrm{~d}$ gauge couplings proceeds as above, thus leading to a scaling compatible with the WCC.

- In addition to D3-brane charged particles, there are 5d membranes of real codimension 2, which implement monodromies associated to the discrete group elements. As in the abelian case, these objects are charged under a dual discrete gauge symmetry (this can be made more manifest by introducing non-harmonic forms to represent the torsion classes $[22,49])$. However, since these objects are not charged under any continuous symmetry, we lack a good handle to constrain their properties, and we will not discuss them further.

The $\mathbf{Z}_{\boldsymbol{k}}$ distance conjectures. It is interesting to explore the relation between the $\mathbf{Z}_{k}$ WCC and the AdS Distance Conjecture in the present setup where, using (3.6), going to large $k$ implies going to large $R$. This is a decompactification limit (note that the orbifold only reduces lengths in $\mathbf{S}^{5}$ in some directions, so the KK scale remains $R^{-1}$ ), in which also the AdS cosmological constant goes to zero, approaching flat space. Hence we can apply 
the AdS Distance Conjecture, which e.g. in its strong version (as we have supersymmetry) establishes that there should be a tower of states with masses scaling as

$$
m_{\mathrm{tw}} \sim \frac{1}{R} \sim M_{p, 5} N^{-\frac{2}{3}} k^{-\frac{1}{3}}
$$

where we have also kept the dependence on $N$. From the $1 / R$ dependence, it is clear the tower corresponds to KK modes. These are the familiar particles dual to single trace chiral primary mesonic operators of the dual SCFT, extensively studied in the literature [50], see [51]. Note that, even though the scaling with $k$ is the same as for wrapped D3-branes, KK modes are lighter due to the relative factor of $N$.

A further subtlety. The above discussion has overlooked an important subtlety. The discrete symmetry $\mathbf{Z}_{k}$ (in fact the full discrete Heisenberg group) is intertwined with the $\mathrm{U}(1)$ in the following sense. Since the D3-branes are charged under the U(1) with charge $N$, a set of $k$ D3-branes carries no discrete $\mathbf{Z}_{k}$ charge, but carries $k N$ units of momentum and cannot decay to the vacuum. In fact, the instanton processes removing the discrete $\mathbf{Z}_{k}$ charge (which correspond to a D3-brane wrapped on the 4-chain whose boundary is $k$ times the torsion 3-cycle) produce simultaneously $N$ particles each carrying momentum $k$ on the circle (whose radius is $R / k$ due to the orbifold).

The situation is very analogous to the one we will encounter in M-theory and type IIA compactifications in section 4 , so we postpone the discussion. Suffice it to say that in this kind of situation, the actual discrete symmetry has order $k^{2}+N^{2}$, heuristically corresponding to the fact that the discrete charge may be eliminated via emission of $k$ D3-branes (each with charge $k$ under the discrete group) and $N$ KK modes (each with charge $N$ under the discrete group). In the regime where the gravity description of $\mathbf{S}^{5} / \mathbf{Z}_{k}$ is valid, we need large $R^{4} \sim N k$ and large $R / k \sim N^{1 / 4} k^{-3 / 4}$, hence $N \gg k^{3}$, and the order of the gauge group is effectively dominated by the $N^{2}$ term, corresponding to emission of $N$ KK modes. Hence, the actual discrete symmetry in this regime is an effective $\mathbf{Z}_{N}$.

It is straightforward to repeat the above computations for the KK mode particles. The mass is given by $k / R$, as corresponds to mesonic operators of dimension $k$ (or multiples of it) due to the orbifold action. We obtain the relations and scalings

$$
m \sim M_{p, 5} N^{-\frac{2}{3}} k^{\frac{2}{3}} \quad, \quad m \sim g k M_{p, 5}^{3 / 2} \quad, \quad g M_{p, 5}^{\frac{1}{2}}=N^{-\frac{2}{3}} k^{-\frac{1}{3}} .
$$

Here $g$ is obviously the same as in (3.14), but we repeat it for convenience. Happily, it is clear that $g$ obeys a $\mathbf{Z}_{N}$ WCC. Notice also that the discretely charged KK modes fit more nicely with the black hole argument in section 2.1. It seems more manageable to emit KK particles than D3-brane particles, as the later extend to a very large size in the internal dimension.

As anticipated, we will re-encounter a very similar situation in M-theory compactifications in the next section, with the additional handle of a type IIA reduction which makes these aspects far more intuitive. We refer the reader to those sections for details. 


\section{M-theory orbifolds and ABJM}

In this section we study the WCC in M-theory on $\mathrm{AdS}_{4} \times \mathbf{S}^{7} / \mathbf{Z}_{k}$ and its type IIA reduction, which provide the gravity dual of the ABJM gauge theories [32]. These theories display interesting new subtleties as compared with earlier cases. Some have been partially discussed in the ABJM literature, so we can again profit from the holographic dictionary.

\subsection{M-theory on $\operatorname{AdS}_{4} \times \mathrm{S}^{7} / \mathrm{Z}_{k}$}

Let us now consider M-theory on $\mathrm{AdS}_{4} \times \mathbf{S}^{7} / \mathbf{Z}_{k}$, where $\mathbf{Z}_{k}$ is generated by $z_{i} \rightarrow e^{2 \pi i / k} z_{i}$ in the underlying $\mathbf{C}^{4}$. This theory is the dual to the ABJM theories, which correspond to $\mathrm{U}(N)_{k} \times \mathrm{U}(N)_{-k}$ Chern-Simons matter theories, ${ }^{4}$ with $\pm k$ denoting the CS level.

The curvature radius of the covering $\mathbf{S}^{7}$ and the $\mathrm{AdS}_{4}$ are given by

$$
R^{6}=2^{5} \pi^{2} M_{p, 11}^{-6} N k
$$

where the factor of $N k$ is analogous to that in section 3 .

We are interested in studying gauge symmetries in the $4 \mathrm{~d}$ theory. The $4 \mathrm{~d}$ Planck scale is given by

$$
M_{p, 4}^{2}=\frac{M_{p, 11}^{9} R^{7}}{k}
$$

Hence we have

$$
R \sim M_{p, 11}^{-1} N^{\frac{1}{6}} k^{\frac{1}{6}}
$$

and then

$$
M_{p, 11} \sim M_{p, 4} N^{-\frac{7}{12}} k^{-\frac{1}{12}} \quad, \quad R \sim M_{p, 4}^{-1} N^{\frac{3}{4}} k^{\frac{1}{4}}
$$

There are two relevant symmetries. There is a $\mathrm{U}(1)$ isometry, surviving from the underlying isometry of $\mathbf{S}^{7}$ which decomposes as $\mathrm{SO}(8) \rightarrow \mathrm{SU}(4) \times \mathrm{U}(1)$ under the orbifold action $z_{i} \rightarrow e^{2 \pi i / k} z_{i}$. It is a continuous gauge symmetry in $\mathrm{AdS}_{4}$. In addition, the internal space has a non-trivial torsion group $H_{5}\left(\mathbf{S}^{7} / \mathbf{Z}_{k}\right)=\mathbf{Z}_{k}$ which allows to obtain $4 \mathrm{~d}$ particles by wrapping M5-branes on the torsion 5-cycle. In the covering space the minimal charge particle is essentially an M5-brane giant graviton, similar to those in the $\operatorname{AdS}_{4} \times \mathbf{S}^{7}$ theory. In particular, it carries $N$ units of momentum on the $\mathbf{S}^{1}$ associated to the $\mathrm{U}(1)$ symmetry.

This seems a perfect candidate for a WGC particle charged under the discrete symmetry, so we consider its properties, in analogy with the D3-brane particles in section 3. Its mass is given by

$$
m_{\mathrm{M} 5} \sim \frac{M_{p, 11}^{6} R^{5}}{k}=M_{p, 4} N^{\frac{1}{4}} k^{-\frac{1}{4}}
$$

\footnotetext{
${ }^{4}$ Actually, as mentioned below and pointed out in [32] the global structure is different such that there are gauge invariant dibaryons for arbitrary $N, k$.
} 
where, in the last equation, we have used (4.4). Note that we recover the AdS/CFT dictionary relation

$$
m_{\mathrm{M} 5}=\frac{N}{R},
$$

indicating that the M5-brane particle is dual to an operator of conformal dimension $N$, as befits a dibaryon.

We can compare this mass with the WGC bound (BPS bound), by computing the gauge coupling. This is just given by the KK reduction of the 11d Einstein terms and gives

$$
g^{-2} \sim M_{p, 11}^{9}\left(R^{7} k^{-1}\right) R^{2} .
$$

Note that we have taken the normalization factor $R^{2}$, which holds when $\operatorname{gcd}(N, k)=1$. This is because in that normalization, the charges under the $\mathrm{U}(1)$ are KK modes of momentum multiple of $k$ (since the radius is $R / k$ due to the orbifold action), and M5-branes, whose charges are multiples of $N$. Then by Bezout's lemma, the minimal charge quantum is 1. For the general case $\operatorname{gcd}(N, k)=r$, we would have a factor $(R / r)^{2}$. We proceed with the coprime case in what follows. As pointed out in [32], the existence of gauge invariant dibaryon operators for general $N$ (not a multiple of $k$ ) implies a specific choice of the global structure of the gauge group of the holographically dual ABJM field theory, see footnote 4 .

Using (4.4) we have

$$
g^{-2} \sim N^{\frac{3}{2}} k^{\frac{1}{2}} \rightarrow g \sim N^{-\frac{3}{4}} k^{-\frac{1}{4}} .
$$

So we get the WGC/BPS relation

$$
m_{\mathrm{M} 5}=M_{p, 4} g N
$$

It is interesting that in the large $k$ limit we recover a weak coupling scaling result $g \sim k^{-1 / 4}$, but that this decrease is slower than the critical $g \sim k^{-1 / 2}$ required by the black hole evaporation argument. The resolution of this point reveals two interesting related subtleties: the actual discrete gauge symmetry of the theory is not just $\mathbf{Z}_{k}$, and the wrapped M5-branes are not the only states charged under the discrete symmetry. Indeed, as mentioned in [32], a set of $k$ wrapped M5-brane particles can unwrap, but they do not decay to the vacuum, but rather turn into $N$ KK states with momentum along the $\mathrm{U}(1)$ circle (which, due to the $\mathbf{Z}_{k}$ orbifold, is quantized in multiples of $k$ ). In other words, there are instantons (given by M5-branes wrapped on the $\mathbf{C P}^{3}$ base of the Hopf fibration of $\mathbf{S}^{7} / \mathbf{Z}_{k}$ ), which emit $k$ M5-branes and $N$ minimal momentum KK modes. As will be more intuitively explained in section 4.2 , there is a discrete symmetry of order $N^{2}+k^{2}$, under which a wrapped M5-brane has charge $k$ and a minimal momentum KK mode has charge $N$. Thus KK modes provide a possible alternative to allow for black hole decay, which in fact is dominated by processes of emission of $N$ such KK modes. Hence, the gauge coupling needs to obey a WCC with respect to $N$. Let us thus check this point.

The KK particle mass is given by

$$
m_{\mathrm{KK}}=\frac{k}{R} .
$$


This in fact constitutes the holographic dictionary relation for an operator of conformal dimension $k$. These are constructed with $k$ copies of a bifundamental field, as required by gauge invariance under the level- $k \mathrm{U}(1)$ 's of the holographic dual field theory [32].

Using (4.4) we have

$$
m_{\mathrm{KK}}=M_{p, 4} N^{-\frac{3}{4}} k^{\frac{3}{4}}
$$

and with (4.8) we obtain

$$
m_{\mathrm{KK}}=M_{p, 4} g k .
$$

Hence these are WGC particles charged under the discrete symmetry, and the gauge coupling (4.8) obeys a WCC bound with respect to $N$.

\subsection{Type IIA description of ABJM vacua}

We may now describe the type IIA version of the previous section, which makes some of the above points more intuitive, and also provides a good warm-up for coming sections.

The type IIA limit arises as follows. The $\mathbf{S}^{7}$ is a $\mathbf{S}^{1}$ Hopf fibration over $\mathbf{C P}^{3}$, where the $\mathbf{Z}_{k}$ quotient acts on the $\mathbf{S}^{1}$. The radius of the $\mathbf{C P}^{3}$ factor is large whenever $N k \gg 1$. From (4.1) we conclude that the M-theory description is valid whenever $k^{5} \ll N$. When $k$ increases, we end up in a weakly coupled regime and we can reduce to type IIA string theory [32].

The type IIA background corresponds to a compactification on $\mathrm{AdS}_{4} \times \mathbf{C P}^{3}$ with internal and AdS radii $R_{s}$ (see below), with $N$ units of $F_{6}$ RR flux over $\mathbf{C P}^{3}$ (i.e. of $F_{4}$ flux over $\mathrm{AdS}_{4}$ ) and $k$ units of $F_{2}$ RR flux over $\mathbf{C P}^{1} \subset \mathbf{C P}^{3}$ (due to the Hopf fibration of the M-theory $\mathbf{S}^{1}$ ).

The matching of string theory quantities to the $11 \mathrm{~d}$ Planck scale is as follows. The 10d string coupling $g_{s}$ is related to the M-theory radius $\mathcal{R}=R / k$ as

$$
g_{s}=M_{p, 11}^{3 / 2} \mathcal{R}^{3 / 2}
$$

that scales as

$$
g_{s} \sim N^{\frac{1}{4}} k^{-\frac{5}{4}} .
$$

The string scale $M_{s}$ is related to the $11 d$ Planck scale as

$$
M_{p, 11}^{3}=\frac{M_{s}^{3}}{g_{s}} .
$$

So in terms of $M_{s}$, the radius (4.1) becomes

$$
R \sim N^{\frac{1}{6}} k^{\frac{1}{6}} g_{s}^{\frac{1}{3}} M_{s}^{-1} .
$$

Finally we need the radius $R_{s}$ of $\mathbf{C P}^{3}$ from the string viewpoint. The type IIA metric is given by

$$
d s_{I I A}^{2}=R_{s}^{2}\left(\frac{1}{4} d s_{\mathrm{AdS}_{4}}^{2}+d s_{\mathbf{C P}^{3}}^{2}\right),
$$


where

$$
R_{s}^{2} \sim N^{1 / 2} k^{-1 / 2} M_{s}^{-2} .
$$

We can now compute the $4 \mathrm{~d}$ Planck mass:

$$
M_{p, 4}^{4} \sim M_{s}^{8} g_{s}^{-2} R_{s}^{6}
$$

and combine with (4.18), (4.19) and (4.14) to obtain

$$
R_{s} \sim N k^{-1} M_{p, 4}^{-1} g_{s}^{-1} \sim M_{p, 4}^{-1} N^{\frac{3}{4}} k^{\frac{1}{4}}, \quad M_{s} \sim N^{-3 / 4} k^{3 / 4} M_{p, 4} g_{s} \sim M_{p, 4} N^{-\frac{1}{2}} k^{-\frac{1}{2}} .
$$

Let us now consider the gauge symmetries in the $4 \mathrm{~d}$ theory in this type IIA string compactification. The SU(4) symmetry arises as the isometry of the internal $\mathbf{C P}^{3}$. On the other hand, there are additional U(1) gauge fields arising from the 10d RR fields, concretely the 10d RR 1-form potential and the 10d RR 3-form potential integrated over $\mathbf{C P}^{1} \subset \mathbf{C P}^{3}$. We should however notice that there are Stückelberg couplings arising from the 10d Chern-Simons coupling $B_{2} F_{2} F_{6}$, of the form ${ }^{5}$

$$
N B_{2} F_{2}+k B_{2} F_{2}^{\prime}
$$

where $F_{2}^{\prime}=\int_{\mathbf{C P}^{2}} F_{6}$. This implies that the massless $\mathrm{U}(1)$ linear combination is

$$
J=k Q_{0}-N Q_{4} .
$$

Here the generators $Q_{0}, Q_{4}$ are labeled by the objects charged under the corresponding U(1)'s, namely D0-branes and D4-branes wrapped on $\mathbf{C P}^{2}$. Note that our sign convention differs from [32].

The orthogonal linear combination,

$$
Q_{\text {broken }}=N Q_{0}+k Q_{4},
$$

corresponds to a massive $\mathrm{U}(1)$, which is broken by instanton effects, and only a discrete subgroup remains. The instanton corresponds to an NS5-brane wrapped on $\mathbf{C P}^{3}$, since it couples magnetically to $B_{2}$. It suffers from Freed-Witten anomalies due to the $F_{6}$ and $F_{2}$ fluxes, so it emits $N$ D0-branes and $k$ wrapped D4-branes. Hence, the total violation of $Q_{\text {broken }}$ is $N^{2}+k^{2}$. This is the order of the gauge group. However, notice that at the level of the black hole (and of the WCC), what is actually relevant is the number of particles required to be emitted, namely $N$ D0-branes (contributing charge $N$ each) and $k$ D4-branes (contributing charge $k$ each). The type IIA internal space is large compared with the string scale if $N \gg k$, so the limit of large order of the discrete gauge group scales as $N^{2}$ and the black hole decay is dominated by the emission of $N$ D0-branes. In the arguments below, this is one particular instance in which the relevant coefficient in scaling relations is not the order of the discrete symmetry, but the number of emitted particles.

Notice also that we are recovering in possibly more intuitive terms the discussion of the earlier M-theory setup, with wrapped D4-branes corresponding to wrapped M5-branes and D0-branes corresponding to KK modes of the M-theory circle.

\footnotetext{
${ }^{5}$ For further discussion of Chern-Simons couplings and swampland constraints see [52].
} 
Let us discuss the masses of the D4- and D0-brane particles and the U(1) gauge couplings. They scale as

$$
\begin{aligned}
& m_{\mathrm{D} 0}=g_{s}^{-1} M_{s} \sim M_{p, 4} N^{-\frac{3}{4}} k^{\frac{3}{4}}, \\
& m_{\mathrm{D} 4}=g_{s}^{-1} M_{s}^{5} R_{s}^{4} \sim M_{p, 4} N^{\frac{1}{4}} k^{-\frac{1}{4}} .
\end{aligned}
$$

We already notice that the D0-brane mass decreases with $N$ faster than the 'species' bound reviewed in section 2.1.1, ensuring that black holes can get rid of their discrete charge by emitting D0-branes. Let us turn to check the implication for gauge couplings and verify the $\mathbf{Z}_{N}$ WCC.

The $4 \mathrm{~d}$ gauge couplings for the $\mathrm{U}(1)$ 's generated by $Q_{0}$ and $Q_{4}$ are given by

$$
\begin{aligned}
& \frac{1}{g_{0}^{2}} \sim M_{s}^{8} R_{s}^{6} M_{s}^{-2} \\
& \frac{1}{g_{4}^{2}} \sim M_{s}^{8} R_{s}^{6}\left(M_{s}^{-5} R_{s}^{-4}\right)^{-2} .
\end{aligned}
$$

The first common factor arises from the reduction of the $10 \mathrm{~d}$ kinetic term for RR fields on the $\mathbf{C P}^{3}$, while the last factors arise from the normalization of the gauge fields by the coefficient of the D-brane Chern-Simons term, so that charges are integer numbers. Using the familiar relations above, we obtain the scalings

$$
g_{0}^{-2} \sim N^{3 / 2} k^{-3 / 2} \quad, \quad g_{4}^{-2} \sim N^{-1 / 2} k^{1 / 2} .
$$

The coupling constant associated to the massless combination (4.22) is

$$
g^{-2}=\frac{k^{2}}{g_{0}^{2}}+\frac{N^{2}}{g_{4}^{2}} \sim N^{\frac{3}{2}} k^{\frac{1}{2}}
$$

and, as explained, its scaling satisfies the WCC with respect to $N$

$$
g \sim N^{-\frac{3}{4}} k^{-\frac{1}{4}} .
$$

As expected, the D0- and D4-brane particles satisfy the BPS/WGC bound, in agreement with the result for wrapped M5-branes and KK modes in (4.9), (4.12)

$$
m_{\mathrm{D} 4}=M_{p, 4} g N \quad, \quad m_{\mathrm{D} 0}=M_{p, 4} g k .
$$

Notice also that $g \sim 1 / R$ in Planck units, so the above masses imply conformal dimensions $N$ and $k$ for the holographically dual operators, as is by now familiar.

\section{Discrete 3-form symmetries and scale separation in AdS solutions}

In [34] it is proposed that in AdS vacua with cosmological constant $\Lambda$, the limit $\Lambda \rightarrow 0$ is accompanied by a tower of states becoming light as

$$
m \sim|\Lambda|^{\alpha}
$$


The strong version of this conjecture is that $\alpha=1 / 2$, which is the case in many/most string solutions (see below for examples). We focus on this version and phrase the conjecture as a ratio of scales ${ }^{6}$

$$
\frac{m^{2}}{\Lambda} \sim \mathcal{O}(1)
$$

The states in the tower are typically KK states, and we use this term in the following. The conjecture implies that one cannot achieve a (parametric) separation of the KK scale and the scale of the cosmological constant. In fact, a problem that has been pervasive in holography literature is the search of gravity duals of QCD or 4d SCFT with conformal anomaly coefficients $a \neq c$. Scale separation is also an important intermediate step in constructions attempting to realize de Sitter vacua in string theory $[53,54]$. Hence it is an important question which merits attention.

There are systematic constructions of $\mathrm{AdS}_{4}$ vacua in string theory in type IIA compactifications on CY orientifolds with NSNS and RR fluxes [33, 55] (see [56] for a recent generalization to general CYs). As already noticed in the literature, there is a family of vacua in [33] (see also [55]) claimed to achieve scale separation, thus violating the strong form of the conjecture. In this section we show that this family enjoys a $\mathbf{Z}_{k}$ discrete symmetry arising from 3 -form gauge symmetries broken by a topological coupling to an axion, of the kind considered in $[35,36]$, together with a continuous 3 -form symmetry. Hence it provides a setup in which a $\mathbf{Z}_{k}$ WCC for 3 -form gauge fields is at work. The tension of the corresponding BPS domain walls can be related to the vacuum energy, and introduces additional factors of $k$ in (5.2), thus explaining the parametric scale separation, that is controlled by the parameter $k$. This symmetry is consistently absent in other AdS vacua with no scale separation, hence provides a rationale for the existence of scale separation in this family, and suggests the proper generalization of (5.2) in the presence of domain wall $\mathbf{Z}_{k}$ symmetries.

\subsection{Review of scaling $\mathrm{AdS}_{4}$ vacua with scale separation}

In this section we review some key elements of the family of models with scale separation, following [33] (see also [55] for related classes of type IIA AdS vacua).

Consider type IIA on a CY threefold modded out by an orientifold action introducing O6-planes. The O6-planes introduce a tadpole for the RR 7-form, which is canceled by (possibly present) D6-branes, and a combination of the $F_{0} \equiv m$ Romans mass flux parameter and $H_{3}$ NSNS field strength flux on 3-cycles. Although it is possible to introduce it, we consider the RR $F_{2}$ field strength fluxes to be zero. ${ }^{7}$ On the other hand, we introduce RR $F_{4}$ field strength fluxes on a basis of 4 -cycles $\tilde{\Sigma}_{i}$

$$
\int_{\tilde{\Sigma}_{i}} F_{4}=e_{\tilde{i}} \in \mathbf{Z} \text {. }
$$

\footnotetext{
${ }^{6}$ Note that $\Lambda$ has dimension mass ${ }^{2}$.

${ }^{7}$ Actually, by monodromies in suitable axions [24] the $F_{2}$ flux can be generated due to the presence of $F_{0}$ flux. This follows from a Dvali-Kaloper-Sorbo coupling, and intertwines non-trivially with similar DKS coupling to appear in section 5.2. We keep our simplified discussion for $F_{2}=0$, and refer the reader to $[57,58]$ for further information on the more general framework.
} 
We do not introduce RR $F_{6}$ flux over the CY, and only consider it when generated by monodromies, see section 5.3. Some details on the $4 \mathrm{~d}$ effective action of this theory are provided in appendix $\mathrm{C}$, and here we streamline the key facts. Whereas the fluxes $F_{0}=m$ and $H_{3}$ are constrained to be $\mathcal{O}(1)$ due to the tadpole conditions, the fluxes for $F_{4}$ are unconstrained and can be taken large. The scaling solutions are achieved in the large $k$ limit of

$$
e_{\tilde{i}} \sim \bar{e}_{\tilde{i}} k,
$$

where the $\bar{e}_{\tilde{i}}$ are $\mathcal{O}(1)$ quantities. Note that we have renamed the scaling parameter of [33] as $k$ to make better contact with earlier sections, and to emphasize its forthcoming role as related to a discrete gauge symmetry.

Although we keep much of the upcoming discussion general, it is useful to consider explicit examples. A simple class is obtained by taking toroidal orbifolds $\mathbf{T}^{6} / \mathbf{Z}_{3}$, whose untwisted sector is given by 3 Kähler moduli associated to the 3 underlying $\mathbf{T}^{2}$ 's. Their volumes, measured in string units, are denoted by $v_{i}, i=1,2,3$, with the overall volume being $\overline{\mathcal{V}} \sim v_{1} v_{2} v_{3}$. They are complexified by the axions from the NSNS 2-form over the 2 -tori $b_{i}$. We ignore twisted sectors, and refer the reader to [33] for details. Since $h_{2,1}=0$, there is only one axion $\xi$ from the period of the RR 3-form over the 3-cycle; it combines with the $4 \mathrm{~d}$ dilaton $e^{D}$ to form a complex modulus.

In the scaling limit, [33] found a supersymmetric $\mathrm{AdS}_{4}$ minimum (which we refer to as the DGKT solution) with the following values for the $4 \mathrm{~d}$ moduli

$$
v_{i}, b_{i} \sim k^{\frac{1}{2}} \quad, \quad \overline{\mathcal{V}} \sim k^{\frac{3}{2}}, \quad e^{-D}, \xi \sim k^{\frac{3}{2}} .
$$

This implies that

$$
M_{s}^{2} \sim e^{2 D} M_{p, 4}^{2} \sim k^{-3} M_{p, 4}^{2},
$$

and that the following relevant quantities of the $4 \mathrm{~d}$ effective action, evaluated at the minimum, and measured in 4d Planck units, scale as

$$
W \sim k^{\frac{3}{2}} \quad, \quad e^{\mathcal{K}} \sim k^{-\frac{15}{2}} \quad \Lambda \sim k^{-\frac{9}{2}} .
$$

One may evaluate the KK scale as

$$
m_{\mathrm{KK}} \sim \overline{\mathcal{V}}^{-\frac{1}{6}} M_{s} \sim k^{-\frac{7}{4}} M_{p, 4}
$$

(incidentally, it coincides with the mass scale for other massive moduli, so it provides a general cutoff of the $4 \mathrm{~d}$ theory).

This leads to a relation of the type (5.1)

$$
m_{\mathrm{KK}}^{2} \sim \Lambda^{\frac{7}{9}},
$$

and hence to a seeming parametric violation of the strong version of the conjecture. In [59] the problem was considered in a family of IIA compactifications with geometric fluxes. The back-reaction of the latter [60] implied a modification of $m_{\mathrm{KK}}$ which restored the 
scaling predicted by the strong AdS Distance Conjecture. This mechanism however is not obviously available in the present context, where geometric moduli are absent. In the following sections we propose the scale separation is physical in these cases, and find a rationale in terms of underlying symmetries.

\subsection{The discrete 3-form symmetry}

In this section we address the backbone of the solution to the above conundrum. First, notice that we had rewritten the strong conjecture as in the form (5.2) with hindsight. Indeed, taking this ratio we find that in the DGKT family

$$
\frac{m_{\mathrm{KK}}^{2}}{\Lambda} \sim k
$$

Alternatively, we may express the vacuum energy $\Lambda$ in terms of the UV cutoff scale $m_{\mathrm{KK}}$ as

$$
\Lambda \sim \frac{m_{\mathrm{KK}}^{2}}{k} .
$$

Recalling that $\Lambda$ has dimension 2, this is extremely reminiscent of the type of relation one finds in theories with a $\mathbf{Z}_{k}$ discrete gauge symmetry, see (2.3). Moreover, since the left hand side quantity is the vacuum energy, the relevant charged objects should be related to the structure of the vacuum.

We now show that there is indeed an effective $\mathbf{Z}_{k}$ symmetry acting on domain walls changing the fluxes in the vacuum. The structure is controlled by topological couplings of the $10 \mathrm{~d}$ theory. In fact, we will study them without assuming the vacuum solution described in the previous section, and show that the scaling relations found there are a consequence of these topological couplings, or equivalently of the discrete symmetry structure.

So we start with the general CY (orientifold) compactification, and consider the basis of 4-cycles $\tilde{\Sigma}_{i}$ and their dual 2-cycles $\Sigma_{i}$. We recall the $F_{4}$ flux structure and introduce $4 \mathrm{~d}$ axions from $B_{2}$ as

$$
\int_{\tilde{\Sigma}_{i}} F_{4}=k \bar{e}_{\tilde{i}} \quad, \quad \int_{\Sigma_{i}} B_{2}=\phi_{i}
$$

(these axions were denoted by $b_{i}$ in the toroidal setup above). In addition, we introduce a symplectic basis of orientifold-odd 3-cycles $\alpha_{a}$ and orientifold-even 3 -cycles $\beta_{a}$, and introduce the NSNS $H_{3}$ fluxes and RR axions

$$
\int_{\alpha_{a}} H_{3}=p_{a} \quad, \quad \int_{\beta_{a}} C_{3}=\xi_{a} .
$$

In addition, there is a Romans mass flux parameter $F_{0}=m$.

Let us initially focus on the dynamics of Kähler moduli, hence ignore $\xi_{a}$, which will be reintroduced later on. Most of the discussion is general, although we eventually apply it to the toroidal orbifold for illustration.

The dimensional reduction of the $10 \mathrm{~d}$ Chern-Simons coupling $F_{4} F_{4} B_{2}$ leads to the $4 \mathrm{~d}$ topological coupling

$$
k\left(\sum_{i} \bar{e}_{\tilde{i}} \phi_{i}\right) F_{4}
$$


This makes the 3 -form massive, by eating up the 2-form dual to a linear combination of axions. The overall factor $k$ implies that there is a discrete $\mathbf{Z}_{k}$ symmetry under which domain walls are charged [21]. This confirms we are on the right track. In fact, although certain modifications are about to come in, in the large $k$ limit this $\mathbf{Z}_{k}$ discrete symmetry determines the properties of the system.

The situation is actually slightly more subtle, because of the following. The scalars $\phi_{i}$ also appear in couplings with other 4-forms, arising from the 8-form as

$$
F_{4, \tilde{i}}=\int_{\tilde{\Sigma}_{i}} F_{8}
$$

Hence, including the reduction of the $10 \mathrm{~d}$ coupling $F_{0} B_{2} F_{8}$, the complete set of topological couplings is

$$
m \sum_{i} \phi_{i} F_{4, \tilde{i}}+k\left(\sum_{i} \bar{e}_{\tilde{i}} \phi_{i}\right) F_{4}
$$

This means that the combination $\phi^{\prime} \equiv \sum_{i} \bar{e}_{\tilde{i}} \phi_{i}$ also couples to other 4 -forms. To isolate that dependence, introduce the generators $Q^{\prime}$ and $Q_{i}$ of 3 -form U(1) symmetries for $C_{3}$ and $C_{3, i}$, and consider the linear combination

$$
Q^{\prime}=\sum_{i} \bar{e}_{\tilde{i}} Q_{i}
$$

The topological coupling for the corresponding 4 -form $F_{4}^{\prime}$ is

$$
m\left(\sum_{i} \bar{e}_{\tilde{i}} \phi_{i}\right) F_{4}^{\prime}=m \phi^{\prime} F_{4}^{\prime} .
$$

Hence, we can isolate the axion $\phi^{\prime}$ with its couplings to the 4 -forms $F_{4}, F_{4}^{\prime}$ as

$$
\phi^{\prime}\left(m F_{4}^{\prime}+k F_{4}\right) \text {. }
$$

It is interesting that we have this universal sector, decoupled (at the topological level) from other axions and 4-forms, and hence independent of the details of the underlying CY compactification space.

Since there is only one axion and two 4 -forms, there is clearly a massless 3 -form corresponding to the combination

$$
Q_{\mathrm{U}(1)}=k Q^{\prime}-m Q=\sum_{i} e_{\tilde{i}} Q_{\tilde{i}}-m Q
$$

In the second equality we have recast the combination in terms of the original 4 -forms. It is straightforward to check, using (5.16), that $Q_{\mathrm{U}(1)}$ is indeed free from topological couplings to scalars, hence remains an unbroken 3-form gauge symmetry.

The combination appearing in (5.19), namely

$$
Q_{\perp}=m Q^{\prime}+k Q=\sum_{i} m \bar{e}_{\tilde{i}} Q_{\tilde{i}}+k Q
$$


is broken to a discrete subgroup. To better understand its structure, consider the string emitting a number of domain walls, and let us compute the violation of conservation of $Q_{\perp}$. The relevant string couples to the dual to $\phi$, namely it is given by an NS5-brane wrapped on the linear combination of 4-cycles $\sum_{i} \bar{e}_{\tilde{i}} \tilde{\Sigma}_{i}$. Due to the presence of $m$, it emits $m \bar{e}_{\tilde{i}}$ D6-branes wrapped on $\tilde{\Sigma}_{i}$; due to the presence of $e_{\tilde{i}}$ units of 4 -form flux over $\tilde{\Sigma}_{i}$, it emits $\sum_{i} \bar{e}_{\tilde{i}} e_{\tilde{i}}$ D2-branes. Since each D6-brane on $\tilde{\Sigma}_{i}$ violates $Q_{\tilde{i}}$ in 1 unit, and each D2-brane violates $Q$ in 1 unit, we have a total violation of $Q_{\text {broken }}$ by

$$
\Delta Q_{\perp}=\sum_{i}\left(\bar{e}_{\tilde{i}}\right)^{2}\left(k^{2}+m^{2}\right)
$$

Although it would seem that at large $k$ the symmetry is of order $k^{2}$, notice that it suffices to have $k$ D2-branes (plus a number of D6's sub-leading in the $1 / k$ approximation) to annihilate into a string. It's only that one D2-brane implies a violation of $k$ units of $Q_{\mathrm{broken}}$, from the way we built the linear combination. So it is an effective $\mathbf{Z}_{k}$ for D2-branes.

Notice that this system realizes a 3 -form version of the theories with discrete and continuous $\mathrm{U}(1)$ symmetries (for 1-forms) we described in earlier sections. In particular, the structure of two underlying U(1)'s with one linear combination broken by a topological coupling is completely analogous to the discussion of the type IIA gravity dual of ABJM theories in section $4.2 .^{8}$

\subsection{Scaling relations for moduli from discrete symmetries}

In analogy with the ABJM system, the D2- and D6-brane domain walls are BPS, and their tensions must relate to their charges under the unbroken $Q_{\mathrm{U}(1)}$,

$$
T_{\mathrm{DW}}=g Q_{\mathrm{U}(1)} M_{p, 4}^{4} \text {. }
$$

The gauge coupling $g$ for $Q_{\mathrm{U}(1)}$ is derived from those of the 3 -form symmetries associated to $Q$ and $Q_{\tilde{i}}$, see (5.20). We denote them $g_{2}, g_{6, \tilde{i}}$ respectively, to indicate that the charged objects are D2-branes and D6-branes on $\Sigma_{\tilde{i}}$. We have

$$
\frac{1}{g^{2}}=k^{2} \sum_{i}\left(\bar{e}_{\tilde{i}}\right)^{2} \frac{1}{g_{6, \tilde{i}}^{2}}+m^{2} \frac{1}{g_{2}^{2}} .
$$

The fact that both D2- and D6-branes can satisfy the BPS condition (5.23), implies that, in the large $k$ limit, their gauge couplings must relate as

$$
g_{6, \tilde{i}} \sim k g_{2} .
$$

It is easy to express the ratio of these gauge couplings in terms of microscopic compactification parameters and derive that the scaling for $v$ reproduces (5.5). We offer a simplified discussion here, referring the reader to appendix $\mathrm{C}$ for a supergravity-friendly derivation. For concreteness, we also focus on the toroidal case. The inverse gauge couplings squared are

$$
\begin{aligned}
& \frac{1}{g_{2}^{2}}=M_{s}^{2} \overline{\mathcal{V}}\left(M_{s}^{-3}\right)^{2}=M_{s}^{-4} \overline{\mathcal{V}}, \\
& \frac{1}{g_{6, \tilde{i}}^{2}}=M_{s}^{2} \overline{\mathcal{V}}\left(M_{s}^{-3} \frac{v_{i}}{\overline{\mathcal{V}}}\right)^{2}=M_{s}^{-4} \frac{\left(v_{i}\right)^{2}}{\overline{\mathcal{V}}},
\end{aligned}
$$

\footnotetext{
${ }^{8}$ With the notational difference that the roles of $N, k$ are now played by $k, m$, respectively.
} 
where the first factor arises from the 10d coupling and the terms in parenthesis arise from normalization of charges to integers, and we recall that $\overline{\mathcal{V}}=v_{1} v_{2} v_{3}$. We have that

$$
\frac{g_{6, \tilde{i}}}{g_{2}}=\frac{\overline{\mathcal{V}}}{v_{i}}
$$

and comparing with (5.25) for different $i$ 's gives

$$
v_{i} \sim k^{\frac{1}{2}}, \overline{\mathcal{V}} \sim k^{\frac{3}{2}} .
$$

A more direct, and possibly more general, route to the scaling relations for moduli is to use the monodromy relations. The fact that e.g. $F_{4}$ has topological couplings to axions implies that the flux $N$ of $F_{6}$ over the CY changes as the axions wind across their periods. Indeed, the above discussion is slightly oversimplified, since the fluxes experience a more intricate set of axion monodromies. These have been studied systematically in [57], and appeared implicitly in [33]. They just follow from the nested structure of 10d Chern-Simons terms, or equivalently of the $10 \mathrm{~d}$ modified Bianchi identity for $F_{6}$, which implies

$$
F_{6}=d C_{5}+F_{4} B_{2}+F_{2} B_{2} B_{2}+F_{0} B_{2} B_{2} B_{2}+H_{3} C_{3} .
$$

Hence, restricting to our setup with only $F_{0}, F_{4}$ and $H_{3}$, the effective 4 d theory can depend only on the combination

$$
N+k \bar{e}_{\tilde{i}} \phi_{i}+m \kappa_{i j k} \phi_{i} \phi_{j} \phi_{k}+p_{a} \xi_{a}
$$

(where sums over repeated indices are implicit). Here $\kappa_{i j k}$ is the triple intersection number. For instance, $\kappa_{123}=1$ for the torus. This implies that it is possible to generate $F_{6}$ flux from $m$ by performing a monodromy in $b_{1}$ to generate $F_{2}$ on the first $\mathbf{T}^{2}$, followed by a monodromy in $b_{2}$ to generate $F_{4}$ on the $\mathbf{T}^{4}$ transverse to the third coordinate, and one in $b_{3}$ to generate $F_{6}$ on the $\mathrm{CY}$.

This is a more complete version of the topological couplings to 4 -forms we have been considering, and which underlies the discrete symmetry of the system. We are interested in its behavior in the large $k$ limit. Consistent scaling of the monodromy relations for large $k$ requires that

$$
\phi_{i} \sim k^{\frac{1}{2}}
$$

This is the generalization of the scaling for $b_{i}$ in (5.5), and provides the complexified counterpart of our scalings for $v_{i}$ in (5.28) (which recovered those in (5.5)). We point out that the fact that the two components of complex moduli have identical scalings with large flux quanta fits nicely with results on asymptotic flux compactification [61]. It is extremely interesting that this result follow from just the discrete symmetry in the present context.

Motivated by this, we can use a similar argument to extract the scaling of the dilaton multiplet in the large $k$ limit. From (5.30) we get

$$
\xi_{a} \sim k^{\frac{3}{2}} .
$$


This is the complexification of a similar dependence of the dilaton, which thus reproduces (5.5).

Interestingly, with this information, which in particular implies the scaling (5.6), i.e. $M_{s} \sim k^{-3 / 2} M_{p, 4}$, we obtain the scaling of gauge couplings (5.26), (5.24)

$$
g_{2} \sim k^{-\frac{15}{4}} \quad, \quad g_{6, \tilde{i}} \sim k^{-\frac{11}{4}} \quad, \quad g \sim k^{-\frac{15}{4}},
$$

providing a nice version of the WCC for domain walls.

Note however that when including the $H_{3}$ fluxes, the above discussion is equivalent to the inclusion of additional topological couplings $p_{a} \xi_{a} F_{4}$. In other words, D2-brane domain walls, in the presence of $H_{3}$ flux, can annihilate in sets of $p_{a}$ by nucleating a string given by a D4-brane wrapped on the 3-cycle $\alpha_{a}$, due to the Freed-Witten inconsistency of the latter. The presence of these couplings spoils the structure of continuous and discrete $3-$ form gauge symmetries found in the Kähler moduli sector. In other words, the coupling of $F_{4}$ to a different linear combination of axions implies that the former continuous symmetry is actually also broken by the new additional axion, given by the linear combination of $\xi_{a}$. We skip the detailed discussion of the resulting complete discrete symmetry group. Note however that for large $k$ the effects of both $m$ and $p$ are sub-leading in a $1 / k$ expansion, so the $\mathbf{Z}_{k}$ symmetry we have been using prevails.

Since we have recovered the scalings of the Kähler and complex structure moduli, it is a simple exercise to use the expressions of $4 \mathrm{~d}$ supergravity to derive others like (5.7), and eventually recover the scale separation (5.11). On the other hand, the $4 \mathrm{~d}$ approach has been criticized as potentially hiding subtleties of the $10 \mathrm{~d}$ solution. Therefore in the following we use an alternative approach, and exploit properties of BPS domain walls to recover the vacuum energy.

\subsection{Discrete symmetries and scale separation}

In this section we exploit the interplay between the tensions of domain walls and the vacuum energy, and study the interplay of discrete symmetries and scale separation. We argue through explicit examples that AdS vacua with trivial discrete symmetry for domain walls do not have scale separation; this is true even if there are non-trivial discrete symmetries for particles or strings, and in general for real codimension higher than 1 objects. On the other hand, we show that the above type IIA modes with non-trivial discrete symmetry for domain walls, with the corresponding scaling for moduli, do have vacuum energy with scale separation. We extend this general relation and put forward the following refined version of the swampland constraint (5.2), as follows:

$\mathbf{Z}_{k}$ refined strong ads distance conjecture. Consider quantum gravity on an AdS vacuum with a $\mathbf{Z}_{k}$ discrete symmetry for domain walls (with $k$ large). In the flat-space limit $\Lambda \rightarrow 0$ (with $\Lambda k \rightarrow 0$ as well) there exists an infinite tower of states at a scale $M_{\text {cutoff }}$, with the relation

$$
\Lambda \sim \frac{M_{\text {cutoff }}^{2} .}{k} .
$$

We now proceed to check this conjecture in the examples of supersymmetric AdS vacua of this paper, by deriving their vacuum energies from the properties of domain walls. 


\subsubsection{Vacuum energy from domain walls}

Let us describe our main tool to evaluate the vacuum energies without invoking an underlying scalar potential. There is in fact a general relation between domain wall tensions and vacuum energies, which essentially follows from junction conditions in general relativity. We refer the reader to appendix D for a discussion well adapted to our application in AdS. The key point is that the domain wall tension $T$ is the variation of certain quantities $\lambda$, see (D.9), whose square essentially gives the vacuum energy $\Lambda$, see (D.8). In the supersymmetric setup, and for BPS domain walls, these statements become the familiar

$$
\lambda=e^{\mathcal{K} / 2} W \quad, \quad T=\Delta\left(e^{\mathcal{K} / 2} W\right)=\Delta \lambda \quad, \quad \Lambda=-3 e^{\mathcal{K}}|W|^{2} \sim-|\lambda|^{2} .
$$

We consider BPS domain walls whose quantized charge describes the change in some field strength flux $n$ as one crosses the domain wall. In the limit of large flux $n$, the tension $T$ provides the derivative of $d \lambda / d n$. We can then solve to obtain the scaling with $n$ of $\lambda$, and thus of its square, $\Lambda$.

\subsubsection{Warm-up examples: no scale separation}

We now turn to discuss the AdS examples of sections 3, 4, deriving their AdS radius from the above strategy, and showing there is no scale separation. This is in agreement with our Refined Strong AdS Distance Conjecture (RSADC), as these examples have discrete symmetries for particles (and for their dual real codimension 2 objects) but not for domain walls.

Type IIB on $\mathbf{S}^{\mathbf{5}} / \mathbf{Z}_{\boldsymbol{k}}$. Consider type IIB on $\mathbf{S}^{5} / \mathbf{Z}_{k}$ with $N$ units of RR 5 -form flux and

$$
R^{4} \sim M_{s}^{-4} g_{s} N k .
$$

This is of course the class of theories considered in section 3, but we are now not imposing the solution for the $5 \mathrm{~d}$ vacuum, rather we are deriving its vacuum energy from the domain wall properties. In passing, we also discuss the gauge coupling of the 3 -forms and draw conclusions regarding the WCC.

We consider a BPS domain wall given by a D3-brane in $5 \mathrm{~d}$. Its tension is

$$
T_{\mathrm{D} 3} \sim M_{s}^{4} g_{s}^{-1} \sim M_{p, 5}^{4} N^{-\frac{5}{3}} k^{-\frac{1}{3}} .
$$

The same result is obtained from the BPS condition

$$
T_{\mathrm{D} 3}=g Q_{\mathrm{D} 3}
$$

upon computation of the gauge coupling of the 5d RR 4-form under which the D3-brane is charged. Since the tension essentially agrees with the gauge coupling, we observe an interesting WCC scaling for $g$ (in that respect, recall that the relevant large order discrete symmetry is $\mathbf{Z}_{N}$ ). This is interesting, since the discrete symmetry acts on particles/membranes, whereas $g$ is a 3 -form gauge coupling. It would be interesting to explore the interplay between discrete and continuous symmetries of different degrees; we hope to come back to this in future work. 
Since this domain wall interpolates among vacua with $N$ and $N+1$, one can now obtain

$$
\frac{d \lambda}{d N} \sim N^{-\frac{5}{3}} k^{-\frac{1}{3}} \Rightarrow \lambda \sim N^{-\frac{2}{3}} k^{-\frac{1}{3}} \quad \Rightarrow \quad \Lambda \sim M_{p, 5}^{2} N^{-\frac{4}{3}} k^{-\frac{2}{3}} .
$$

Using (3.6) we have

$$
\Lambda \sim R^{-2}
$$

Hence the AdS radius is the same as that of the internal space, and there is no decoupling of scales. This is the strong ADC statement in [34].

Note that, even though there are discrete gauge symmetries in the system, their orders do not enter the ratio of scales. This is in agreement with our RSADC, since these discrete symmetries involve particles and membranes, not domain walls.

M-theory on $\mathbf{S}^{\mathbf{7}} / \mathbf{Z}_{\boldsymbol{k}}$. Let us consider M-theory on $\mathbf{S}^{7} / \mathbf{Z}_{k}$ with $N$ units of flux (or $N k$ in the covering space) and

$$
R^{6} \sim M_{p, 11}^{-6} N k
$$

This is of course the same system as in section 4, but again we wish to derive the $4 \mathrm{~d}$ vacuum energy from the relevant BPS domain walls. We consider a BPS domain wall given by an M2-brane in $4 \mathrm{~d}$. Its tension is

$$
T_{\mathrm{M} 2} \sim M_{p, 11}^{3} \sim M_{p, 4}^{3} N^{-\frac{7}{4}} k^{-\frac{1}{4}} .
$$

where we used (4.4). The same result is obtained from the BPS condition

$$
T_{\mathrm{M} 2}=g Q_{\mathrm{M} 2}
$$

upon computation of the gauge coupling $g$ for the $4 \mathrm{~d} 3$-form. Recalling the relevant large order discrete symmetry is $\mathbf{Z}_{N}$, we note again that we get an interesting WCC scaling for $g$.

Since the M2-brane domain wall interpolates between vacua with $N$ and $N+1$ units of flux, we have

$$
\frac{d \lambda}{d N} \sim N^{-\frac{7}{4}} k^{-\frac{1}{4}} \Rightarrow \lambda \sim N^{-\frac{3}{4}} k^{-\frac{1}{4}} \Rightarrow \Lambda \sim M_{p, 4}^{2} N^{-\frac{3}{2}} k^{-\frac{1}{2}} \sim R^{-2} .
$$

In the last relation, we have used (4.4). Again, we recover the result that the AdS radius is of the same order of magnitude as the KK scale of the internal space. Also, notice that there are discrete symmetries in the theory, but they involve particles and strings, rather than domain walls. Hence, they do not alter the relation between scales, in agreement with our RSADC.

Type IIA on $\mathbf{C P}^{3}$. We would like to repeat the previous computation in the type IIA picture. Let us consider type IIA theory on $\mathbf{C P}^{3}$ with $N$ units of $F_{6}$ RR flux over $\mathbf{C P}^{3}$ and $k$ units of $F_{2}$ RR flux over $\mathbf{C P}^{1} \subset \mathbf{C P}^{3}$ and

$$
R_{s}^{2} \sim M_{s}^{-2} N^{1 / 2} k^{-1 / 2} .
$$


This is the same system as in section 4.2. The relevant BPS domain wall is a D2-brane in $4 \mathrm{~d}$, whose tension is

$$
T_{\mathrm{D} 2} \sim M_{s}^{3} g_{s}^{-1} \sim M_{p, 4}^{3} N^{-7 / 4} k^{-1 / 4} .
$$

This is the same scaling as the M2-brane in the previous section, and the D2-brane domain wall interpolates vacua with $N$ and $N+1$ units of flux, so we recover

$$
\Lambda \sim M_{p, 4}^{2} N^{-3 / 2} k^{-1 / 2} \sim R_{s}^{-2} .
$$

The AdS radius is the same as that of the internal space, with no scale separation, in agreement with our RSADC.

\subsubsection{Revisiting the scale separation in type IIA CY flux compactifications}

Consider now the configurations with the large $k$ discrete $\mathbf{Z}_{k}$ symmetry for domain walls in section 5.2. We wish to derive the scaling of the vacuum energy with $k$, just using the scaling of moduli vevs (5.5), (5.6) derived in section 5.3 from the $\mathbf{Z}_{k}$ symmetry.

We consider the BPS domains wall given by a D4-brane wrapped on the combination of 2-cycles $\sum_{i} \bar{e}_{\tilde{i}} \Sigma_{i}$. This domain wall interpolates between vacua with $F_{4}$ flux given by $k$ and $k+1$. Notice that the $F_{4}$-flux is not monodromic, hence the D4-branes are stable against nucleation of strings, and can provide BPS objects (in contrast with e.g. D2- and D6-brane domain walls encountered in earlier sections).

The tension of these domain walls can be obtained from the BPS equation and the gauge couplings, computed in detail in appendix C. Here we carry out a simplified derivation, taking the toroidal case for concreteness. The gauge coupling of a $\mathrm{D} 4_{i}$-brane domain wall is

$$
\frac{1}{g_{4, i}^{2}}=M_{s}^{2} \overline{\mathcal{V}}\left(M_{s}^{-3} v_{i}^{-1}\right)^{2}=M_{s}^{-4} \overline{\mathcal{V}} v_{i}^{-2} \sim k^{\frac{13}{2}}
$$

As usual, in the first equality, the first term comes from the reduction of the 10d coupling, and the parenthesis from the charge normalization. Note that the scaling is common for all $i$, so by the BPS condition we get the tension

$$
T_{\mathrm{DW}} \sim k^{-\frac{13}{4}} .
$$

Notice that, if interpreted in terms of gauge couplings, this implies an interesting WCC, as in earlier examples. From the above tension we get

$$
\frac{d \lambda}{d k} \sim k^{-\frac{13}{4}} \Rightarrow \lambda \sim k^{-\frac{9}{4}} \Rightarrow \Lambda \sim k^{-\frac{9}{2}} .
$$

So we recover the scaling (5.7) for $\Lambda$ (the reader can check those of $\mathcal{K}$ and $W$ as well). Once $m_{\mathrm{KK}}$ is recovered as in (5.8), this reproduces the scale separation (5.11), in agreement with our RSADC conjecture.

\section{Acknowledgments}

We are pleased to thank L. Ibáñez, F. Marchesano for useful discussions. This work is supported by the Spanish Research Agency (Agencia Española de Investigación) through 
the grant IFT Centro de Excelencia Severo Ochoa SEV-2016-0597, and the grant FPA201565480-P from the MCU/AEI/FEDER. The work by J.C. is supported by a FPU position from Spanish Ministry of Education. A.M. received funding from "la Caixa" Foundation (ID 100010434) with fellowship code LCF/BQ/IN18/11660045 and from the European Union's Horizon 2020 research and innovation programme under the Marie SkłodowskaCurie grant agreement No. 713673.

\section{A Species bound for extremal black holes}

In the following we consider the evaporation of extremal black holes endowed with $\mathbf{Z}_{k}$ charge. For concreteness, the classical solutions we are taking are the extremal ReissnerNordström black holes in $4 \mathrm{~d}$ space-time dimensions. They have vanishing Hawking temperature, so the analysis in [41] is not directly applicable.

Extremal black holes can discharge through Schwinger radiation [62-64]. Whenever the electric field is much larger than the background curvature, this happens essentially in flat space [65]. In this case the production rate has an exponential suppression

$$
\Gamma \sim e^{-\frac{m^{2}}{q E}} \sim e^{-\chi}
$$

where $m$ and $q$ are the mass and charge of the emitted particle and $E$ is the electric field, given by

$$
E=\frac{g^{2}}{4 \pi} \frac{Q}{r^{2}} .
$$

As argued in section 2.1.2, the simplest way in which this kind of black hole is able to get rid of both continuous and discrete charge while remaining sub-extremal is in the presence of a $\mathbf{Z}_{k}$ WGC particle. Let us assume that this particle is actually BPS,

$$
m=g q M_{p} .
$$

As a consequence, the black hole will remain extremal throughout the whole evaporation process.

From (A.1) and (A.2), we notice that the maximum particle production will happen close to the horizon, so in this order of magnitude analysis we will approximate the whole radiation as the contribution of that region.

From the extremality condition we can relate the horizon radius and the charge of the $\mathrm{BH}$ with its mass through

$$
r_{h} \sim \frac{M_{\mathrm{BH}}}{M_{p}^{2}}, \quad g Q \sim \frac{M_{\mathrm{BH}}}{M_{p}} .
$$

They lead to

$$
E \sim g \frac{M_{p}^{3}}{M_{\mathrm{BH}}} .
$$

Introducing (A.3) and (A.5) in (A.1) we can estimate the factor in the exponential suppression of the production rate of the $\mathbf{Z}_{k}$ WGC particle to be

$$
\chi \sim \frac{m M_{\mathrm{BH}}}{M_{p}^{2}} .
$$


The black hole will be able to efficiently evaporate discrete charge when

$$
M_{\mathrm{BH}} \lesssim \frac{M_{p}^{2}}{m}
$$

With this condition being true, the black hole should still have enough mass to radiate $\mathcal{O}(k)$ particles (assuming the $\mathbf{Z}_{k}$ WGC particle to have unit discrete charge), which means

$$
M_{\mathrm{BH}} \gtrsim k m \text {. }
$$

Finally, from the two conditions (A.7) and (A.8), we obtain the following bound for the mass of the $\mathbf{Z}_{k}$ WGC particle:

$$
m^{2} \lesssim \frac{M_{p}^{2}}{k}
$$

This is the species bound in [41]. We have shown that the bound also applies to extremal black holes emitting $\mathbf{Z}_{k}$ WGC particles via Schwinger effect.

\section{B Discrete symmetries in intersecting brane models}

Discrete symmetries are ubiquitous in models of intersecting branes (see [66] for a review), as pioneered in [20]. In this appendix we use them to illustrate the interplay of $\mathbf{Z}_{k}$ and $\mathrm{U}(1)$ gauge symmetries, and the scalings implied by the $\mathbf{Z}_{k}$ WCC.

Let us start by recalling the basic setup. Consider a compactification of type IIA on a Calabi-Yau space $\mathbf{X}_{6}$ quotiented by the orientifold ${ }^{9}$ action $\Omega \mathcal{R}(-1)^{F_{L}}$, where $\mathcal{R}$ is an antiholomorphic $\mathbf{Z}_{2}$ involution of $\mathbf{X}_{6}$, which introduces O6-planes. Let us denote $\left[\Pi_{\mathrm{O} 6}\right]$ the total homology class of the 3-cycles wrapped by the O6-planes. Introducing a symplectic basis $\left[\alpha_{i}\right],\left[\beta_{i}\right]$ of 3 -cycles even and odd under $\mathcal{R}$, respectively, we may expand

$$
\left[\Pi_{\mathrm{O} 6}\right]=\sum_{i} r_{\mathrm{O} 6}^{i}\left[\alpha_{i}\right]+s_{\mathrm{O} 6}^{i}\left[\beta_{i}\right]
$$

with $r_{\mathrm{O} 6}^{i}, s_{\mathrm{O} 6}^{i}$ some coefficients of order 1-10.

The O6-planes are charged under the RR 7-form, so to cancel its tadpoles we introduce D6-branes. We consider stacks of $N_{A}$ overlapping $\mathrm{D} 6{ }_{A}$-branes wrapped on 3 -cycles $\Pi_{A}$, and their orientifold image D6 $A^{\prime}$-branes on 3 -cycles $\Pi_{A^{\prime}}$. In terms of the basis, we have

$$
\left[\Pi_{A}\right]=\sum_{i} r_{A}^{i}\left[\alpha_{i}\right]+s_{A}^{i}\left[\beta_{i}\right] \quad, \quad\left[\Pi_{A^{\prime}}\right]=\sum_{i} r_{A}^{i}\left[\alpha_{i}\right]-s_{A}^{i}\left[\beta_{i}\right] .
$$

The RR tadpole condition reads

$$
\sum_{A} 2 r_{A}^{i}+r_{\mathrm{O} 6}^{i}=0 \quad \forall i
$$

In addition there are K-theory RR tadpole conditions [67], which we skip in this sketchy discussion.

\footnotetext{
${ }^{9}$ Note that the orientifolds are not essential for the argument, but we choose to introduce them to better connect with the literature on intersecting brane models.
} 
In these models, there are Stückelberg couplings for the $\mathrm{U}(1)_{A}$, of the form

$$
\sum_{A} N_{A} s_{A}^{i} b_{2, i} F_{A}
$$

where wedge product is implicit. $F_{A}$ is the field strength of the $\mathrm{U}(1)$ gauge field on the D $6_{A}$-branes, and the $4 \mathrm{~d} 2$-forms $b_{2, i}$ arise from the KK compactification of the RR 5 -form $C_{5}$ as

$$
b_{2, i}=\int_{\beta_{i}} C_{5} .
$$

This makes some of the U(1)'s massive. Let us consider linear combinations of the $\mathrm{U}(1)_{A}$ generators $Q_{A}$

$$
Q=\sum_{A} c_{A} Q_{A}
$$

with $c_{A}$ being coprime integers, so as to preserve charge integrality. The Stückelberg coupling for the field strength $F$ of the $\mathrm{U}(1)$ generated by $A$ is

$$
\left(\sum_{A} c_{A} N_{A} s_{A}^{i}\right) b_{2, i} F
$$

Hence, the condition for a $\mathrm{U}(1)$ to remain massless is

$$
\sum_{A} c_{A} N_{A} s_{A}^{i}=0 \quad \forall i
$$

If not, the $\mathrm{U}(1)$ is broken, remaining only as approximate global symmetry, broken by nonperturbative D2-brane instanton effects [68-70]. The condition that a discrete $\mathbf{Z}_{k}$ subgroup remains as exact discrete gauge symmetry is

$$
\sum_{A} c_{A} N_{A} s_{A}^{i}=0 \bmod k \quad \forall i
$$

Generically, to achieve this for large $k$ a possibility ${ }^{10}$ is to have $s_{A}^{i} \sim k$, at least for some $A$, for all $i$. This implies that there is some brane which is wrapped on a very large (i.e. multiply wrapped) cycle. This implies that in general any unbroken $\mathrm{U}(1)$, given by a linear combination (B.6) satisfying (B.8), will also involve that particular $Q_{A}$ with a coefficient of order $k$. This implies that the gauge coupling of the unbroken $\mathrm{U}(1)$ scales as

$$
\frac{1}{g^{2}}=k \quad \text { hence } g \sim k^{-\frac{1}{2}},
$$

in agreement with the $\mathbf{Z}_{k}$ WCC.

Although this is not quite a rigorous argument, it is a good illustration of how the interplay between $\mathrm{U}(1)$ gauge couplings and $\mathbf{Z}_{k}$ symmetries arises, as a consequence of the fact that, to achieve a large order $\mathbf{Z}_{k}$ discrete symmetry, one needs to use parametrically large cycles, thus parametrically scaling gauge couplings to zero. Hence, intersecting brane models provide an intuitive mechanism for the $\mathbf{Z}_{k}$ WCC. More detailed string theory examples are presented in the main text.

\footnotetext{
${ }^{10}$ This is not the only one, but we stick to it as an illustrative example.
} 


\section{Gauge couplings in type IIA CY compactifications}

In this appendix we derive the gauge coupling constants for domain walls present in type IIA CY flux compactifications. We review the computation in [71] following the conventions in [33]. From [33], the 10d string frame action is given by ${ }^{11}$

$$
\begin{aligned}
S^{10 d}=\frac{1}{2 \kappa_{10}^{2}} \int d^{10} x \sqrt{-g}\left(e ^ { - 2 \phi } \left(R+4\left(\partial_{\mu} \phi\right)^{2}\right.\right. & \left.-\frac{1}{2}\left|H_{3}^{\text {total }}\right|^{2}\right) \\
& \left.-\left(\left|\tilde{F}_{2}\right|^{2}+\left|\tilde{F}_{4}\right|^{2}+m_{0}^{2}\right)\right)+S_{\mathrm{CS}},
\end{aligned}
$$

where $2 \kappa_{10}^{2}=(2 \pi)^{7} \alpha^{\prime 4}$ and the definitions of the field strengths are

$$
\begin{aligned}
H_{3}^{\text {total }} & =d B_{s}+H_{3}^{\mathrm{bg}}, \\
\tilde{F}_{2} & =d C_{1}+m B_{2}, \\
\tilde{F}_{4} & =d C_{3}+F_{4}^{\mathrm{bg}}-C_{1} \wedge H_{3}-\frac{m}{2} B_{2} \wedge B_{2} .
\end{aligned}
$$

The Chern-Simons action contains also a prefactor $\left(2 \kappa_{10}\right)^{-1}$ in front. We define an adimensional internal volume by $\overline{\mathcal{V}}=M_{s}^{6} \mathcal{V}$ and perform the dimensional reduction in the string frame. For instance, the kinetic term for the $4 \mathrm{~d}$ field strength associated to the $10 \mathrm{~d}$ $F_{4}$ reads

$$
S_{4 d}^{\text {kin }} \supset-\frac{M_{s}^{2}}{2} \int d_{4} x \sqrt{-g_{4}} \overline{\mathcal{V}}\left|\mathcal{F}_{4}\right|^{2},
$$

where $\mathcal{F}_{4}=d C_{3}$. To move back to the Einstein frame, we choose a reference scale $a$, and define the 4 d dilaton $D(x)$ as

$$
a=\frac{\langle\overline{\mathcal{V}}\rangle}{e^{2\langle\phi\rangle}} \quad, \quad e^{2 D}=\frac{e^{2 \phi}}{\overline{\mathcal{V}}} .
$$

So the Einstein frame kinetic terms take the form

$$
S_{E}^{\mathrm{kin}} \supset \frac{M_{s}^{2}}{2 a} \int d^{4} x \sqrt{-g_{E}} R_{E}-\frac{a^{2} M_{s}^{2}}{2} \int d^{4} x \sqrt{-g_{E}} \overline{\mathcal{V}} e^{-4 D}\left|\mathcal{F}_{4}\right|^{2},
$$

where the products are now done using $g_{E}$ as a metric.

To obtain $4 \mathrm{~d}$ gauge 3 -forms, we perform a KK reduction of $10 \mathrm{~d}$ p-forms along suitable harmonic ( $\mathrm{p}-3)$-forms in the internal space. In the notation of [57],

$$
C_{3}=c_{3}^{0}, C_{5}=c_{3}^{a} \wedge \omega_{a}, C_{7}=\tilde{d}_{3 a} \wedge \tilde{\omega}^{a} \text { and } C_{9}=\tilde{d}_{3} \wedge \omega_{6} .
$$

They corresponds to the relevant 4 -forms $\mathcal{F}_{4}^{0}, \mathcal{F}_{4}^{a}, \tilde{\mathcal{F}}_{4, a}$ and $\tilde{\mathcal{F}}_{4}$ associated to D2-, D4-, D6and D8-branes.

Notice that we need to normalize the gauge fields by the coefficient in front of the D-brane Chern-Simons term, in order for the charges to be properly quantized. For a Dp-brane this introduces factors of $\mu_{p} \propto \alpha^{\prime(p+1) / 2} \sim M_{s}^{(p+1)}$ in the forthcoming gauge

\footnotetext{
${ }^{11}$ Our convention is that $\left|F_{p}\right|^{2}=F_{\alpha_{1} \ldots \alpha_{p}} F^{\alpha_{1} \ldots \alpha_{p}} / p !$.
} 
couplings. Namely, in order to be consistent, we need to keep the harmonic forms as adimensional, so the generic Chern-Simons action is

$$
S_{\mathrm{CS}}^{(p)} \sim M_{s}^{3} \int_{W_{3} \times \gamma_{p-2}} c_{3} \wedge \omega_{p-2}
$$

where we have called $c_{3} \wedge \omega_{p-2}$ collectively each decomposition in (C.6). The normalization consists in redefining the RR 3-form by a factor $M_{s}^{3}$, so that there is no prefactor in front of the Chern-Simons action. The effect of such redefinition on (C.5) is just a change in the prefactor in front of the kinetic terms of the gauge fields,

$$
S_{E}^{\mathrm{kin}} \supset \frac{M_{s}^{2}}{2 a} \int d^{4} x \sqrt{-g_{E}} R_{E}-\frac{a^{2}}{2 M_{s}^{2}} \int d^{4} x \sqrt{-g_{E}} \overline{\mathcal{V}} e^{-4 D}\left|\mathcal{F}_{4}\right|^{2} .
$$

We are almost done in the definition of the coupling constants, but first we need the following quantities:

$$
M_{s}^{2} \propto \frac{e^{2\langle\phi\rangle}}{\langle\overline{\mathcal{V}}\rangle} M_{p, 4}^{2}=a^{-1} M_{p, 4}^{2}, \quad K_{K}=-\ln (8 \overline{\mathcal{V}}), \quad K_{Q}=4 D, \quad K=K_{K}+K_{Q} .
$$

Substituting in (C.8) and including the other 4-forms, we obtain [71]

$$
S_{E}^{\text {kin }}=\frac{\pi}{2 M_{p}^{4}} \int \frac{e^{-K}}{8}\left[\mathcal{F}_{4}^{0} \wedge \star \mathcal{F}_{4}^{0}+4 g_{a b} \mathcal{F}_{4}^{a} \wedge \star \mathcal{F}_{4}^{b}+\frac{1}{4 \overline{\mathcal{V}}^{2}} g^{a b} \tilde{\mathcal{F}}_{4 \mid a} \wedge \star \tilde{\mathcal{F}}_{4 \mid b}+\frac{1}{\overline{\mathcal{V}}^{2}} \tilde{\mathcal{F}}_{4} \wedge \star \tilde{\mathcal{F}}_{4}\right]
$$

where

$$
g_{a b}=\frac{\partial^{2} K_{K}}{\partial t^{a} \partial \bar{t}^{b}}
$$

is the metric in the Kähler moduli space with $t^{a}=v^{a}+i b^{a}$.

We need now to specialize to the toroidal orbifold in [33]. The Kähler potential is

$$
K_{K}=-\ln \left(8 v^{1} v^{2} v^{3}\right)=-\ln \left(\left(t^{1}+\bar{t}^{1}\right)\left(t^{2}+\vec{t}^{2}\right)\left(t^{3}+\vec{t}^{3}\right)\right),
$$

so

$$
g_{a b}=\frac{1}{4} \operatorname{diag}\left(\left(v^{1}\right)^{-2},\left(v^{2}\right)^{-2},\left(v^{3}\right)^{-2}\right) .
$$

We rewrite the action according to this metric obtaining

$$
\begin{aligned}
S_{E}^{\mathrm{kin}}=\frac{\pi}{2 M_{p}^{4}} \int \frac{e^{-K}}{8}\left[\mathcal{F}_{4}^{0} \wedge \star \mathcal{F}_{4}^{0}+\sum_{i=1}^{3}\left(\frac{1}{\left(v^{i}\right)^{2}} \mathcal{F}_{4}^{i} \wedge \star \mathcal{F}_{4}^{i}+\frac{\left(v^{i}\right)^{2}}{\overline{\mathcal{V}}^{2}} \tilde{\mathcal{F}}_{4 \mid i} \wedge \star \tilde{\mathcal{F}}_{4 \mid i}\right)\right. & \\
& \left.+\frac{1}{\overline{\mathcal{V}}^{2}} \tilde{\mathcal{F}}_{4} \wedge \star \tilde{\mathcal{F}}_{4}\right]
\end{aligned}
$$

We are finally able to read the coupling constants of all kinds of domain walls:

$$
\begin{aligned}
\frac{1}{g_{0}^{2}} & =\frac{\pi e^{-K}}{8 M_{p}^{4}}, & \frac{1}{g_{i}^{2}} & =\frac{\pi e^{-K}}{8 M_{p}^{4}\left(v^{i}\right)^{2}}, \\
\frac{1}{g_{\tilde{i}}^{2}} & =\frac{\pi e^{-K}\left(v^{i}\right)^{2}}{8 M_{p}^{4} \overline{\mathcal{V}}^{2}}, & \frac{1}{g_{\tilde{4}}^{2}} & =\frac{\pi e^{-K}}{8 M_{p}^{4} \overline{\mathcal{V}}^{2}} .
\end{aligned}
$$


From the main text, the scalings with the flux $k$ are

$$
e^{K} \sim k^{-15 / 2}, v_{i} \sim k^{1 / 2} \text { and } \overline{\mathcal{V}} \sim k^{3 / 2},
$$

so the couplings scale as

$$
\begin{aligned}
\frac{1}{g_{0}^{2}} & =\frac{\pi e^{-K}}{8 M_{p}^{4}} \sim k^{15 / 2}, & \frac{1}{g_{i}^{2}} & =\frac{\pi e^{-K}}{8 M_{p}^{4}\left(v^{i}\right)^{2}} \sim k^{13 / 2}, \\
\frac{1}{g_{\tilde{i}}^{2}} & =\frac{\pi e^{-K}\left(v^{i}\right)^{2}}{8 M_{p}^{4} \overline{\mathcal{V}}^{2}} \sim k^{11 / 2} & , & \frac{1}{g_{\tilde{4}}^{2}}=\frac{\pi e^{-K}}{8 M_{p}^{4} \overline{\mathcal{V}}^{2}} \sim k^{9 / 2} .
\end{aligned}
$$

\section{Junction conditions for AdS vacua}

Here we adapt to the $4 \mathrm{~d}$ setup the discussion of [72], which studies a Randall-Sundrum construction [73, 74] with an arbitrary number of branes (domain walls). The discussion is also similar to systems of D8-branes in type I' theory [75].

Consider a 4 d spacetime with $N$ parallel domain walls with tensions $T_{i}$, located at positions $y_{i}$ in a coordinate $y$. The region between the $i^{t h}$ and $(i+1)^{t h}$ brane has cosmological constant $\Lambda_{i}$. A solution of the $4 \mathrm{~d}$ Einstein equations

$$
\begin{aligned}
\sqrt{-G}\left(R_{M N}-\frac{1}{2} G_{M N} R\right)=-\frac{1}{4 M_{p, 4}^{2}}\left[\sum_{i=1}^{N} \Lambda_{i}\left[\theta\left(y-y_{i}\right)-\theta\left(y-y_{i+1}\right)\right] \sqrt{-G} G_{M N}\right. \\
\left.+\sum_{i=1}^{N} T_{i} \sqrt{-g^{(i)}} g_{\mu \nu}^{(i)} \delta_{M}^{\mu} \delta_{N}^{\nu} \delta\left(y-y_{i}\right)\right]
\end{aligned}
$$

is given by the ansatz

$$
d s^{2}=e^{-2 \sigma(y)} \eta_{\mu \nu} d x^{\mu} d x^{\nu}+r_{c}^{2} d y^{2} .
$$

The warp factor in the above expression is given by the following piecewise linear function

$$
\begin{aligned}
\sigma(y)= & \left(\lambda_{1}-\lambda_{0}\right)\left(y-y_{1}\right) \theta\left(y-y_{1}\right)+\left(\lambda_{2}-\lambda_{1}\right)\left(y-y_{2}\right) \theta\left(y-y_{2}\right) \\
& +\ldots+\left(\lambda_{N}-\lambda_{N-1}\right)\left(y-y_{N}\right) \theta\left(y-y_{N}\right)
\end{aligned}
$$

where $\lambda_{0}$ and $\lambda_{N}$ provide the asymptotic behavior at $y \mp \infty$. In any region between two domain walls, we can perform a change of coordinates

$$
\frac{x_{0}}{r_{c}}=e^{\sigma(y)},
$$

to bring the metric (D.2) to a more standard form, i.e.

$$
d s^{2}=\frac{r_{c}^{2}}{x_{0}^{2}}\left(\eta_{\mu \nu} d x^{\mu} d x^{\nu}+d x_{0}^{2}\right) .
$$

from which it is clear that the solution describes slices of $\mathrm{AdS}_{4}$ with different values of the cosmological constant, made explicit below. 
From (D.1), we obtain the following constraints for $\sigma(y)$ [72]:

$$
\begin{aligned}
\left(\sigma^{\prime}(y)\right)^{2} & =-\frac{r_{c}^{2}}{12 M_{p, 4}^{2}} \sum_{i=1}^{N} \Lambda_{i}\left[\theta\left(y-y_{i}\right)-\theta\left(y-y_{i+1}\right)\right], \\
\sigma^{\prime \prime}(y) & =\frac{r_{c}}{8 M_{p, 4}^{2}} \sum_{i=1}^{N} T_{i} \delta\left(y-y_{i}\right) .
\end{aligned}
$$

Substituting (D.3) in (D.6) and (D.7), we obtain the relations

$$
\begin{aligned}
\lambda_{i} & = \pm \sqrt{\frac{-\Lambda_{i} r_{c}^{2}}{12 M_{p, 4}^{2}}}, \\
\frac{T_{i} r_{c}}{8 M_{p, 4}^{2}} & =\lambda_{i}-\lambda_{i-1} .
\end{aligned}
$$

Hence these junction conditions relate the variation of the cosmological constant to the potential of the branes that give us the domain walls. This is a general interpretation of what we proposed in section 5.4.

Open Access. This article is distributed under the terms of the Creative Commons Attribution License (CC-BY 4.0), which permits any use, distribution and reproduction in any medium, provided the original author(s) and source are credited.

\section{References}

[1] C. Vafa, The string landscape and the swampland, hep-th/0509212 [INSPIRE].

[2] H. Ooguri and C. Vafa, On the geometry of the string landscape and the swampland, Nucl. Phys. B 766 (2007) 21 [hep-th/0605264] [INSPIRE].

[3] N. Arkani-Hamed, L. Motl, A. Nicolis and C. Vafa, The string landscape, black holes and gravity as the weakest force, JHEP 06 (2007) 060 [hep-th/0601001] [INSPIRE].

[4] H. Ooguri and C. Vafa, Non-supersymmetric AdS and the swampland, Adv. Theor. Math. Phys. 21 (2017) 1787 [arXiv:1610.01533] [InSPIRE].

[5] G. Obied, H. Ooguri, L. Spodyneiko and C. Vafa, De Sitter space and the swampland, arXiv: 1806.08362 [INSPIRE].

[6] G. Buratti, E. García-Valdecasas and A.M. Uranga, Supersymmetry breaking warped throats and the weak gravity conjecture, JHEP 04 (2019) 111 [arXiv:1810.07673] [INSPIRE].

[7] T.D. Brennan, F. Carta and C. Vafa, The string landscape, the swampland and the missing corner, PoS (TASI2017) 015 [arXiv: 1711.00864] [INSPIRE].

[8] E. Palti, The swampland: introduction and review, Fortsch. Phys. 67 (2019) 1900037 [arXiv: 1903.06239] [INSPIRE].

[9] T. Banks and N. Seiberg, Symmetries and strings in field theory and gravity, Phys. Rev. D 83 (2011) 084019 [arXiv:1011.5120] [INSPIRE].

[10] D. Harlow and H. Ooguri, Constraints on symmetries from holography, Phys. Rev. Lett. 122 (2019) 191601 [arXiv:1810.05337] [INSPIRE]. 
[11] D. Harlow and H. Ooguri, Symmetries in quantum field theory and quantum gravity, arXiv: 1810.05338 [INSPIRE].

[12] N. Craig, I. Garcia Garcia and S. Koren, Discrete gauge symmetries and the weak gravity conjecture, JHEP 05 (2019) 140 [arXiv: 1812.08181] [INSPIRE].

[13] L.M. Krauss and F. Wilczek, Discrete gauge symmetry in continuum theories, Phys. Rev. Lett. 62 (1989) 1221 [INSPIRE].

[14] M.G. Alford and F. Wilczek, Aharonov-Bohm interaction of cosmic strings with matter, Phys. Rev. Lett. 62 (1989) 1071 [INSPIRE].

[15] M.G. Alford, J. March-Russell and F. Wilczek, Discrete quantum hair on black holes and the nonabelian Aharonov-Bohm effect, Nucl. Phys. B 337 (1990) 695 [INSPIRE].

[16] M.G. Alford et al., The interactions and excitations of non-Abelian vortices, Phys. Rev. Lett. 64 (1990) 1632 [Erratum ibid. 65 (1990) 668] [INSPIRE].

[17] M.G. Alford and J. March-Russell, Discrete gauge theories, Int. J. Mod. Phys. B 5 (1991) 2641 [INSPIRE].

[18] M.G. Alford, K.-M. Lee, J. March-Russell and J. Preskill, Quantum field theory of non-Abelian strings and vortices, Nucl. Phys. B 384 (1992) 251 [hep-th/9112038] [INSPIRE].

[19] M.G. Alford, S.R. Coleman and J. March-Russell, Disentangling non-Abelian discrete quantum hair, Nucl. Phys. B 351 (1991) 735 [INSPIRE].

[20] M. Berasaluce-Gonzalez, L.E. Ibáñez, P. Soler and A.M. Uranga, Discrete gauge symmetries in D-brane models, JHEP 12 (2011) 113 [arXiv:1106.4169] [INSPIRE].

[21] M. Berasaluce-Gonzalez, P.G. Camara, F. Marchesano and A.M. Uranga, $Z p$ charged branes in flux compactifications, JHEP 04 (2013) 138 [arXiv: 1211.5317] [INSPIRE].

[22] M. Berasaluce-Gonzalez et al., Non-Abelian discrete gauge symmetries in $4 d$ string models, JHEP 09 (2012) 059 [arXiv: 1206. 2383] [INSPIRE].

[23] L.E. Ibáñez, A.N. Schellekens and A.M. Uranga, Discrete gauge symmetries in discrete MSSM-like orientifolds, Nucl. Phys. B 865 (2012) 509 [arXiv: 1205.5364] [INSPIRE].

[24] F. Marchesano, G. Shiu and A.M. Uranga, F-term axion monodromy inflation, JHEP 09 (2014) 184 [arXiv:1404.3040] [INSPIRE].

[25] M. Montero, A.M. Uranga and I. Valenzuela, Transplanckian axions!?, JHEP 08 (2015) 032 [arXiv: 1503.03886] [INSPIRE].

[26] G. Buratti, J. Calderón and A.M. Uranga, Transplanckian axion monodromy!?, JHEP 05 (2019) 176 [arXiv: 1812.05016] [INSPIRE].

[27] S.R. Coleman, J. Preskill and F. Wilczek, Quantum hair on black holes, Nucl. Phys. B 378 (1992) 175 [hep-th/9201059] [INSPIRE].

[28] I. Garcia Garcia, Properties of discrete black hole hair, JHEP 02 (2019) 117 [arXiv: 1809.03527] [INSPIRE].

[29] S. Gukov, M. Rangamani and E. Witten, Dibaryons, strings and branes in AdS orbifold models, JHEP 12 (1998) 025 [hep-th/9811048] [INSPIRE].

[30] B.A. Burrington, J.T. Liu and L.A. Pando Zayas, Finite Heisenberg groups in quiver gauge theories, Nucl. Phys. B 747 (2006) 436 [hep-th/0602094] [INSPIRE].

[31] E. Garćıa-Valdecasas, A. Mininno and A.M. Uranga, Discrete symmetries in dimer diagrams, JHEP 10 (2019) 091 [arXiv: 1907.06938] [INSPIRE]. 
[32] O. Aharony, O. Bergman, D.L. Jafferis and J. Maldacena, $N=6$ superconformal Chern-Simons-matter theories, M2-branes and their gravity duals, JHEP 10 (2008) 091 [arXiv:0806.1218] [INSPIRE].

[33] O. DeWolfe, A. Giryavets, S. Kachru and W. Taylor, Type IIA moduli stabilization, JHEP 07 (2005) 066 [hep-th/0505160] [INSPIRE].

[34] D. Lüst, E. Palti and C. Vafa, AdS and the swampland, Phys. Lett. B 797 (2019) 134867 [arXiv: 1906.05225] [INSPIRE].

[35] G. Dvali, Three-form gauging of axion symmetries and gravity, hep-th/0507215 [INSPIRE].

[36] N. Kaloper and L. Sorbo, A natural framework for chaotic inflation, Phys. Rev. Lett. 102 (2009) 121301 [arXiv:0811.1989] [INSPIRE].

[37] D. Junghans, O-plane backreaction and scale separation in Type IIA flux vacua, arXiv:2003.06274 [INSPIRE].

[38] F. Marchesano, E. Palti, J. Quirant and A. Tomasiello, On supersymmetric AdS $S_{4}$ orientifold vacua, arXiv: 2003.13578 [INSPIRE].

[39] B. Heidenreich, M. Reece and T. Rudelius, Sharpening the weak gravity conjecture with dimensional reduction, JHEP 02 (2016) 140 [arXiv: 1509.06374] [INSPIRE].

[40] B. Heidenreich, M. Reece and T. Rudelius, Evidence for a sublattice weak gravity conjecture, JHEP 08 (2017) 025 [arXiv: 1606.08437] [INSPIRE].

[41] G. Dvali, Black holes and large $N$ species solution to the hierarchy problem, Fortsch. Phys. 58 (2010) 528 [arXiv:0706.2050] [INSPIRE].

[42] C. Cheung and G.N. Remmen, Naturalness and the weak gravity conjecture, Phys. Rev. Lett. 113 (2014) 051601 [arXiv: 1402.2287] [INSPIRE].

[43] G. Aldazabal et al., Intersecting brane worlds, JHEP 02 (2001) 047 [hep-ph/0011132] [INSPIRE].

[44] G. Aldazabal et al., $D=4$ chiral string compactifications from intersecting branes, J. Math. Phys. 42 (2001) 3103 [hep-th/0011073] [INSPIRE].

[45] B.A. Burrington, J.T. Liu and L.A. Pando Zayas, Central extensions of finite Heisenberg groups in cascading quiver gauge theories, Nucl. Phys. B 749 (2006) 245 [hep-th/0603114] [INSPIRE].

[46] B.A. Burrington, J.T. Liu, M. Mahato and L.A. Pando Zayas, Finite Heisenbeg groups and Seiberg dualities in quiver gauge theories, Nucl. Phys. B 757 (2006) 1 [hep-th/0604092] [INSPIRE].

[47] B.A. Burrington, J.T. Liu and L.A. Pando Zayas, Finite Heisenberg groups from non-Abelian orbifold quiver gauge theories, Nucl. Phys. B 794 (2008) 324 [hep-th/0701028] [INSPIRE].

[48] J. McGreevy, L. Susskind and N. Toumbas, Invasion of the giant gravitons from Anti-de Sitter space, JHEP 06 (2000) 008 [hep-th/0003075] [INSPIRE].

[49] P.G. Camara, L.E. Ibáñez and F. Marchesano, RR photons, JHEP 09 (2011) 110 [arXiv:1106.0060] [INSPIRE].

[50] E. Witten, Anti-de Sitter space and holography, Adv. Theor. Math. Phys. 2 (1998) 253 [hep-th/9802150] [INSPIRE].

[51] O. Aharony et al., Large $N$ field theories, string theory and gravity, Phys. Rept. 323 (2000) 183 [hep-th/9905111] [INSPIRE]. 
[52] M. Montero, A.M. Uranga and I. Valenzuela, A Chern-Simons pandemic, JHEP 07 (2017) 123 [arXiv: 1702.06147] [INSPIRE].

[53] S. Kachru, R. Kallosh, A.D. Linde and S.P. Trivedi, De Sitter vacua in string theory, Phys. Rev. D 68 (2003) 046005 [hep-th/0301240] [INSPIRE].

[54] V. Balasubramanian, P. Berglund, J.P. Conlon and F. Quevedo, Systematics of moduli stabilisation in Calabi-Yau flux compactifications, JHEP 03 (2005) 007 [hep-th/0502058] [INSPIRE].

[55] P.G. Camara, A. Font and L.E. Ibáñez, Fluxes, moduli fixing and MSSM-like vacua in a simple IIA orientifold, JHEP 09 (2005) 013 [hep-th/0506066] [INSPIRE].

[56] F. Marchesano and J. Quirant, A landscape of AdS flux vacua, JHEP 12 (2019) 110 [arXiv: 1908.11386] [INSPIRE].

[57] A. Herraez, L.E. Ibáñez, F. Marchesano and G. Zoccarato, The Type IIA flux potential, 4-forms and Freed-Witten anomalies, JHEP 09 (2018) 018 [arXiv: 1802.05771] [INSPIRE].

[58] D. Escobar, F. Marchesano and W. Staessens, Type IIA Flux Vacua with mobile D6-branes, JHEP 01 (2019) 096 [arXiv: 1811.09282] [INSPIRE].

[59] A. Font, A. Herráez and L.E. Ibáñez, On scale separation in Type II AdS flux vacua, JHEP 03 (2020) 013 [arXiv: 1912.03317] [INSPIRE].

[60] G. Aldazabal and A. Font, A second look at $N=1$ supersymmetric AdS $S_{4}$ vacua of type IIA supergravity, JHEP 02 (2008) 086 [arXiv:0712.1021] [INSPIRE].

[61] T.W. Grimm, C. Li and I. Valenzuela, Asymptotic flux compactifications and the swampland, JHEP 06 (2020) 009 [arXiv: 1910.09549] [INSPIRE].

[62] J. Schwinger, On gauge invariance and vacuum polarization, Phys. Rev. 82 (1951) 664.

[63] W.A. Hiscock and L.D. Weems, Evolution of charged evaporating black holes, Phys. Rev. D 41 (1990) 1142.

[64] G.W. Gibbons, Vacuum polarization and the spontaneous loss of charge by black holes, Cmmoun. Math. Phys. 44 (1975) 245.

[65] M. Montero, T. Van Riet and G. Venken, Festina lente: EFT constraints from charged black hole evaporation in de Sitter, JHEP 01 (2020) 039 [arXiv:1910.01648] [INSPIRE].

[66] L.E. Ibáñez and A.M. Uranga, String theory and particle physics: an introduction to string phenomenology, Cambridge University Press, Cambridge U.K. (2012).

[67] A.M. Uranga, D-brane probes, RR tadpole cancellation and k-theory charge, Nucl. Phys. B 598 (2001) 225 [hep-th/0011048] [INSPIRE].

[68] R. Blumenhagen, M. Cvetič and T. Weigand, Spacetime instanton corrections in $4 D$ string vacua: the seesaw mechanism for D-brane models, Nucl. Phys. B 771 (2007) 113 [hep-th/0609191] [INSPIRE].

[69] L.E. Ibáñez, A.N. Schellekens and A.M. Uranga, Instanton induced neutrino Majorana Masses in CFT orientifolds with MSSM-like spectra, JHEP 06 (2007) 011 [arXiv:0704.1079] [INSPIRE].

[70] B. Florea, S. Kachru, J. McGreevy and N. Saulina, Stringy instantons and quiver gauge theories, JHEP 05 (2007) 024 [hep-th/0610003] [INSPIRE].

[71] A. Font, A. Herráez and L.E. Ibáñez, The swampland distance conjecture and towers of tensionless branes, JHEP 08 (2019) 044 [arXiv: 1904.05379] [INSPIRE]. 
[72] H. Hatanaka, M. Sakamoto, M. Tachibana and K. Takenaga, Many brane extension of the Randall-Sundrum solution, Prog. Theor. Phys. 102 (1999) 1213 [hep-th/9909076] [InSPIRE].

[73] L. Randall and R. Sundrum, A large mass hierarchy from a small extra dimension, Phys. Rev. Lett. 83 (1999) 3370 [hep-ph/9905221] [INSPIRE].

[74] L. Randall and R. Sundrum, An alternative to compactification, Phys. Rev. Lett. 83 (1999) 4690 [hep-th/9906064] [INSPIRE].

[75] J. Polchinski and E. Witten, Evidence for heterotic-Type I string duality, Nucl. Phys. B 460 (1996) 525 [hep-th/9510169] [INSPIRE]. 